\title{
ADAPTIVE BOUNDARY AND POINT CONTROL OF LINEAR STOCHASTIC DISTRIBUTED PARAMETER SYSTEMS*
}

\author{
T. E. DUNCAN ${ }^{\dagger}$, B. MASLOWSKI ${ }^{\ddagger}$, AND B. PASIK-DUNCAN $^{\dagger}$
}

\begin{abstract}
An adaptive control problem for the boundary or the point control of a linear stochastic distributed parameter system is formulated and solved in this paper. The distributed parameter system is modeled by an evolution equation with an infinitesimal generator for an analytic semigroup. Since there is boundary or point control, the linear transformation for the control in the state equation is also an unbounded operator. The unknown parameters in the model appear affinely in both the infinitesimal generator of the semigroup and the linear transformation of the control. Strong consistency is verified for a family of least squares estimates of the unknown parameters. An Itô formula is established for smooth functions of the solution of this linear stochastic distributed parameter system with boundary or point control. The certainty equivalence adaptive control is shown to be self-tuning by using the continuity of the solution of a stationary Riccati equation as a function of parameters in a uniform operator topology. For a quadratic cost functional of the state and the control, the certainty equivalence control is shown to be self-optimizing; that is, the family of average costs converges to the optimal ergodic cost. Some examples of stochastic parabolic problems with boundary control and a structurally damped plate with random loading and point control are described that satisfy the assumptions for the adaptive control problem solved in this paper.
\end{abstract}

Key words. stochastic adaptive control, linear stochastic distributed parameter systems, boundary control problems, identification

AMS subject classifications. 93C40, 93C20, 93E12, 60H15

1. Introduction. An important family of controlled linear, distributed parameter control systems are those with boundary or point control. Perturbations or inaccuracies in the mathematical model can often be effectively modeled by white noise. Since in many control situations there are unknown parameters in these linear, stochastic distributed parameter systems, it is necessary to solve a stochastic adaptive control problem. We now give a brief summary of each of the sections in this paper. In $\S 2$ the unknown linear stochastic distributed parameter system is described by an evolution equation where the unknown parameters appear in the infinitesimal generator of an analytic semigroup and the unbounded linear transformation for the boundary control. The noise process is a cylindrical, white noise. Some properties of the optimal control for the infinite-time quadratic cost functional for the associated deterministic system are reviewed, especially the stationary Riccati equation. These results are given in [8], [11], [12], [18]. In $\S 3$ an Itô formula is obtained for smooth functions of the solution of a linear or semilinear stochastic distributed parameter system with an analytic semigroup. This result is verified using the Yosida approximation of the infinitesimal generator of the semigroup. While some other Itô formulas in infinite dimensions are available (e.g., [6], [15]), none seems to be appropriate for our applications. In $\S 4$ a family of least squares estimates are constructed from the observations of the unknown stochastic system. This family of estimates is shown to be strongly consistent under verifiable conditions. A stochastic differential equation is given for the family of estimates. This verification of the strong consistency of a family of least squares estimates is a generalization of the results in [9], [10]. In $\S 5$ the self-tuning and the self-optimizing properties of an adaptive control law are investigated. If an adaptive control is self-tuning, then it is shown that the system satisfies some stability properties and the adaptive control is self-optimizing. The certainty equivalence adaptive control, that is, using the optimal stationary control with the estimates of the

* Received by the editors April 2, 1992; accepted for publication (in revised form) November 23, 1992. This research was partially supported by National Science Foundation grants ECS-8718026, ECS-9102714, and ECS9113029.

$\dagger$ Department of Mathematics, University of Kansas, Lawrence, Kansas 66045.

$\ddagger$ Institute of Mathematics, Czech Academy of Sciences, Prague, Czech Republic. 
parameters, is shown to be self-optimizing; that is, the optimal ergodic cost is achieved. In $\S 6$ some examples are given that satisfy the various assumptions used in this paper.

2. A boundary control model. The unknown linear stochastic distributed parameter system with boundary or point control is formally described by the following stochastic differential equation:

$$
\begin{aligned}
d X(t ; \alpha) & =(A(\alpha) X(t ; \alpha)+B(\alpha) U(t)) d t+\Phi d W(t), \\
X(0 ; \alpha) & =X_{0},
\end{aligned}
$$

where $X(t ; \alpha) \in H ; H$ is a real, separable, infinite-dimensional Hilbert space; $(W(t), t \geq 0)$ is a cylindrical Wiener process on $H ; \Phi \in \mathcal{L}(H), \alpha=\left(\alpha^{1}, \ldots, \alpha^{q}\right)$; and $t \geq 0$.

The probability space is denoted $(\Omega, \mathcal{F}, P)$, where $P$ is a probability measure that is induced from the cylindrical Wiener measure and $\mathcal{F}$ is the $P$-completion of the Borel $\sigma$-algebra on $\Omega$. Let $\left(\mathcal{F}_{t}, t \geq 0\right)$ be an increasing $P$-complete family of sub- $\sigma$-algebras of $\mathcal{F}$ such that $X_{t}$ is $\mathcal{F}_{t}$-measurable for $t \geq 0$ and $\left(\langle\ell, W(t)\rangle, \mathcal{F}_{t}, t \geq 0\right)$ is a martingale for each $\ell \in H . A(\alpha)$ is the infinitesimal generator of an analytic semigroup on $H$. For some $\beta \geq 0$, the operator $-A(\alpha)+\beta I$ is strictly positive, so that the fractional powers $(-A(\alpha)+\beta I)^{\gamma}$ and $\left(-A(\alpha)^{*}+\beta I\right)^{\gamma}$ and the spaces $D_{A(\alpha)}^{\gamma}=\mathcal{D}\left((-A(\alpha)+\beta I)^{\gamma}\right)$ and $D_{A^{*}(\alpha)}^{\gamma}=\mathcal{D}\left(\left(-A^{*}(\alpha)+\beta I\right)^{\gamma}\right)$ with the graph norm topology for $\gamma \in \mathbb{R}$ can be defined. It is assumed that $B(\alpha) \in \mathcal{L}\left(H_{1}, D_{A(\alpha)}^{\varepsilon-1}\right)$, where $H_{1}$ is a real, separable Hilbert space and $\varepsilon \in(0,1)$ (cf. assumption (A4) below). For the solution of (2.1) on $[0, T]$, the control $(U(t), t \in[0, T])$ is an element of $M_{W}^{p}\left(0, T, H_{1}\right)$, where $M_{W}^{p}\left(0, T, H_{1}\right)=\left\{u:[0, T] \times \Omega \rightarrow H_{1}, u\right.$ is $\left(\mathcal{F}_{t}\right)$ nonanticipative and $\left.E \int_{0}^{T}|u(t)|^{p} d t<\infty\right\}$ and $p>\max (2,1 / \varepsilon)$ is fixed.

A selection of the following assumptions are used subsequently.

(A1) The family of unknown parameters are the elements of a compact set $\mathcal{K}$.

(A2) For $\alpha \in \mathcal{K}$, the operator $\Phi^{*}\left(-A^{*}(\alpha)+\beta I\right)^{-1 / 2+\delta}$ is Hilbert-Schmidt for some $\delta \in\left(0, \frac{1}{2}\right)$.

(A3) There are real numbers $M>0$ and $\omega>0$ such that, for $t>0$ and $\alpha \in \mathcal{K}$,

$$
|S(t ; \alpha)|_{\mathcal{L}(H)} \leq M e^{-\omega t}
$$

and

$$
|A(\alpha) S(t ; \alpha)|_{\mathcal{L}(H)} \leq M t^{-1} e^{-\omega t}
$$

where $(S(t ; \alpha), t \geq 0)$ is the analytic semigroup generated by $A(\alpha)$.

(A4) For all $\alpha_{1}, \alpha_{2} \in \mathcal{K}, \mathcal{D}\left(A\left(\alpha_{1}\right)\right)=\mathcal{D}\left(A\left(\alpha_{2}\right)\right), D_{A\left(\alpha_{1}\right)}^{\delta}=D_{A\left(\alpha_{2}\right)}^{\delta}$ and $D_{A^{*}\left(\alpha_{1}\right)}^{\delta}=$ $D_{A^{*}\left(\alpha_{2}\right)}^{\delta}$ for $\delta \in \mathbb{R}$.

(A5) For each $\alpha \in \mathcal{K}$ and $x \in H$, there is a control $u_{\alpha, x} \in L^{2}\left(\mathbb{R}_{+}, H_{1}\right)$ such that

$$
y(\cdot)=S(\cdot ; \alpha) x+\int_{0} S(\cdot-t ; \alpha) B(\alpha) u_{\alpha, x}(t) d t \in L^{2}\left(\mathbb{R}_{+}, H\right) .
$$

(A6) The operator $A(\alpha)$ has the form

$$
A(\alpha)=F_{0}+\sum_{i=1}^{q} \alpha^{i} F_{i},
$$

where $F_{i}$ is a linear, densely defined operator on $H$ for $i=0,1, \ldots, q$ such that $\cap_{i=0}^{q} \mathcal{D}\left(F_{i}^{*}\right)$ is dense in $H$. 
It is well known that the strong solution of (2.1) may not exist, so usually the mild solution of (2.1) is used, that is,

$$
X(t ; \alpha)=S(t ; \alpha) X_{0}+\int_{0}^{t} S(t-r ; \alpha) B(\alpha) U(r) d r+\int_{0}^{t} S(t-r ; \alpha) \Phi d W(r)
$$

where $S(t ; \alpha)=e^{t A(\alpha)}$. The mild solution is equivalent to the following inner product equation: For each $y \in \mathcal{D}\left(A^{*}(\alpha)\right)$,

$$
\begin{aligned}
\langle y, X(t ; \alpha)\rangle= & \langle y, X(0)\rangle+\int_{0}^{t}\left\langle A^{*}(\alpha) y, X(s ; \alpha)\right\rangle d s \\
& +\int_{0}^{t}\langle\Psi(\alpha) y, U(s)\rangle d s+\left\langle\Phi^{*} y, W(t)\right\rangle,
\end{aligned}
$$

where $\Psi(\alpha)=B^{*}(\alpha) \in \mathcal{L}\left(D_{A^{*}(\alpha)}^{1-\varepsilon}, H_{1}\right)$. The following lemma verifies that $(X(t ; \alpha), t \in$ $[0, T])$ is a well-defined process in $M_{W}^{p}(0, T, H)$.

LEMMA 2.1. Assume that (A2) is satisfied. For $T>0$ and $\alpha \in \mathcal{K}$, the processes $(Z(t ; \alpha), t \in[0, T])$ and $\hat{Z}(t ; \alpha), t \in[0, T])$ given by the equations

$$
\begin{aligned}
& Z(t ; \alpha)=\int_{0}^{t} S(t-r ; \alpha) \Phi d W(r), \\
& \hat{Z}(t ; \alpha)=\int_{0}^{t} S(t-r ; \alpha) B(\alpha) U(r) d r
\end{aligned}
$$

for $U \in M_{W}^{p}\left(0, T, H_{1}\right)$ are elements of $M_{W}^{p}(0, T, H)$, with versions that have continuous sample paths.

Proof. Let $|\cdot|_{H S}$ be the Hilbert-Schmidt norm on $\mathcal{L}(H)$. If $\left(e_{n}\right)$ is an orthonormal basis of $H$, by (A2) we have that

$$
\begin{aligned}
\int_{0}^{T}|S(t ; \alpha) \Phi|_{H S}^{2} d t & \leq \int_{0}^{T} \frac{c}{t^{1-2 \delta}} \sum_{n}\left|(-A(\alpha)+\beta I)^{-1 / 2+\delta} \Phi e_{n}\right|^{2} d t \\
& =\left|(-A(\alpha)+\beta I)^{-1 / 2+\delta} \Phi\right|_{H S}^{2} \int_{0}^{T} \frac{c}{t^{1-2 \delta}} d t<\infty
\end{aligned}
$$

where $c$ is a constant. Thus $(Z(t ; \alpha), t \in[0, T])$ is a well-defined $H$-valued process. To verify the existence of a continuous modification of $(Z(t ; \alpha), t \in[0, T])$, the following processes are introduced:

$$
\tilde{W}(t ; \alpha)=W(t)\left[(-A(\alpha)+\beta I)^{-1 / 2+\delta} \Phi\right]^{*}
$$

and

$$
\tilde{Z}(t ; \alpha)=\int_{0}^{t} S(t-r ; \alpha) d \tilde{W}(r ; \alpha) \quad \text { for } t \in[0, T] .
$$

There is a $D_{A}^{1 / 2-\delta}$-continuous modification of $(\tilde{Z}(t ; \alpha), t \in[0, T])[7$, Thm. 4]. Thus the process

$$
Z(t ; \alpha)=\int_{0}^{t} S(t-r ; \alpha)(-A+\beta I)^{1 / 2-\delta} d \tilde{W}(t ; \alpha)
$$

has an $H$-continuous modification. 
Since the inequality

$$
|S(t-r ; \alpha) B(\alpha)|_{\mathcal{L}\left(H_{1}, H\right)} \leq \frac{c}{(t-r)^{1-\varepsilon}}
$$

is satisfied for $0<r<t<T$, we can apply the Hölder inequality with the exponents $p$ and $q=p /(p-1)$ to the integral $(2.5)$ to verify that $(\hat{Z}(t ; \alpha), t \in[0, T])$ is a well-defined $H$-valued process in $M_{W}^{p}(0, T, H)$ with a continuous modification.

If $A(\alpha)=A^{*}(\alpha)$ and if $(A(\alpha)-\beta I)^{-1}$ is compact, then assumption (A2) is equivalent to the assumption that

$$
\int_{0}^{T} t^{-2 \delta}|S(t ; \alpha) \Phi|_{H S}^{2} d t<\infty
$$

for $T>0$. For notational convenience, the dependence on $\alpha$ is suppressed. By the compactness of the resolvent of $A$, there is a sequence $\left(\lambda_{k}\right)$, where $\lambda_{k}>\lambda_{0}>0$ and $\lambda_{k} \uparrow \infty$, and an orthonormal basis of $\left(e_{k}\right)$ of $H$ such that $(A-\beta I) e_{k}=\lambda_{k} e_{k}$ for $k \in \mathbb{N}$ and

$$
S(t) \cdot=e^{\beta t} \sum_{k} e^{-\lambda_{\kappa} t}<\cdot, e_{k}>e_{k}
$$

If $\tilde{S}(t)=e^{-\beta t} S(t)$, then

$$
\begin{aligned}
\int_{0}^{T} t^{-2 \delta}|\tilde{S}(t) \Phi|_{H S}^{2} d t & =\int_{0}^{T} t^{-2 \delta}\left|\Phi^{*} \tilde{S}(t)\right|_{H S}^{2} d t \\
& =\sum_{k}\left|\Phi^{*} e_{k}\right|^{2} \lambda_{k}^{2 \delta-1} \int_{0}^{\lambda_{k} T} s^{-2 \delta} e^{-2 s} d s \\
& =\sum_{k}\left|\Phi^{*} \lambda_{k}^{-1 / 2+\delta} e_{k}\right|^{2} b_{k}
\end{aligned}
$$

where $0<b_{1} \leq b_{k} \leq b_{k+1}, b_{k} \rightarrow b_{\infty}<\infty$. Since

$$
\sum_{k}\left|\Phi^{*} \lambda_{k}^{-1 / 2+\delta} e_{k}\right|^{2}=\left|\Phi^{*}(-A+\beta I)^{-1 / 2+\delta}\right|_{H S}^{2}
$$

our assertion follows.

Consider the quadratic cost functional

$$
J\left(X_{0}, U, \alpha, T\right)=\int_{0}^{T}[\langle Q X(s), X(s)\rangle+\langle P U(s), U(s)\rangle] d s
$$

where $T \in(0, \infty], X(0)=X_{0}, Q \in \mathcal{L}(H), P \in \mathcal{L}\left(H_{1}\right)$ are selfadjoint operators satisfying

$$
\begin{aligned}
& \langle Q x, x\rangle \geq r_{1}|x|^{2}, \\
& \langle P y, y\rangle \geq r_{2}|y|^{2}
\end{aligned}
$$

for $x \in H, y \in H_{1}$ and constants $r_{1}>0$ and $r_{2}>0$. For the deterministic control problem for (2.1) with $\Phi \equiv 0$ and the cost functional (2.6) with $T=+\infty$ assuming (A5), the optimal cost is $\left\langle V(\alpha) X_{0}, X_{0}\right\rangle$ [8], [12], [18], where $V$ satisfies the formal stationary Riccati equation

$$
A^{*}(\alpha) V(\alpha)+V(\alpha) A(\alpha)-V(\alpha) B(\alpha) P^{-1} \Psi(\alpha) V(\alpha)+Q=0
$$


and $\Psi(\alpha)=B^{*}(\alpha)$.

Equation (2.9) can be modified to a meaningful inner product equation as

$$
\langle A(\alpha) x, V y\rangle+\langle V x, A(\alpha) y\rangle-\left\langle P^{-1} \Psi(\alpha) V x, \Psi(\alpha) V y\right\rangle+\langle Q x, y\rangle=0
$$

for $x, y \in \mathcal{D}(A(\alpha))$. It has been shown [8], [12], [18] that, if (A5) is satisfied, then $V$ is the unique, nonnegative, selfadjoint solution of (2.10) and $V \in \mathcal{L}\left(H, D_{A^{*}}^{1-\varepsilon}\right)$. The solution of (2.9) is understood to be the solution of (2.10).

For adaptive control, the control policies $(U(t), t \geq 0)$ that are considered are linear feedback controls, that is,

$$
U(t)=K(t) X(t)
$$

where $(K(t), t \geq 0)$ is an $\mathcal{L}\left(H, H_{1}\right)$-valued process that is uniformly bounded almost surely by a constant $R>0$. Let $\Delta>0$ be fixed. It is assumed that the $\mathcal{L}\left(H, H_{1}\right)$-valued process $(K(t), t \geq 0)$ has the property that $K(t)$ is adapted to $\sigma(X(u), u \leq t-\Delta)$ for each $t \geq \Delta$. It is also assumed that $(K(t), t \in[0, \Delta])$ is a deterministic, operator-valued function. For such an admissible adaptive control, there is a unique solution of (2.1) with $K(t)=\tilde{K}(X(s), 0 \leq$ $s \leq t-\Delta)$. If $\Delta=0$, then (2.1) may not have a unique solution. Furthermore, the delay $\Delta>0$ accounts for some time that is required to compute the adaptive feedback control law from the observation of the solution of (2.1).

Two more assumptions, (A7) and (A8), are now given that are used for the verification of the strong consistency of a family of least squares estimates of the unknown parameter vector $\alpha$. Define $\mathbb{K} \subset \mathcal{L}\left(H, H_{1}\right)$ as

$$
\mathbb{K}=\left\{K \in \mathcal{L}\left(H, H_{1}\right):|K|_{\mathcal{L}\left(H, H_{1}\right)} \leq R\right\},
$$

where $R$ is given above.

Assume that $B(\alpha)$ is either independent of $\alpha \in \mathcal{K}$ or has the form

$$
B(\alpha)=\Psi^{*}(\alpha)
$$

where $\Psi(\alpha)=\hat{B}^{*} A^{*}(\alpha) \in \mathcal{L}\left(D_{A^{*}(\alpha)}^{1-\varepsilon}, H_{1}\right)$ and the operator $\hat{B} \in \mathcal{L}\left(H_{1}, D_{A(\alpha)}^{\varepsilon}\right)$ is given.

(A7) There is a finite-dimensional projection $\tilde{P}$ on $H$ with range in $\cap_{i=1}^{q} \mathcal{D}\left(F_{i}^{*}\right)$ such that $i_{\tilde{P}} \Phi \Phi^{*} i_{\tilde{P}}^{*}>0$, where $i_{\tilde{P}}: H \rightarrow \tilde{P}(H)$ is the projection map and $B(\alpha)$ is either independent of $\alpha$ or has the form (2.12). In the latter case, there is a finite-dimensional projection $\hat{P}$ on $H$ and a constant $c>0$ such that

$$
\left|\hat{P}\left(I+K^{*} \hat{B}^{*}\right) F^{*} \tilde{P}\right|_{\mathcal{L}(H)}>c
$$

is satisfied for all $F \in\left\{F_{1}, \ldots, F_{q}\right\}$ and $K \in \mathbb{K}$.

It is easy to verify that, if $H$ is infinite-dimensional, if $\hat{B} \in \mathcal{L}\left(H_{1}, H\right)$ is compact, and if $\left(F_{i}^{*}\right)^{-1} \in \mathcal{L}(H)$ for $i=1,2, \ldots, q$, then (A7) is satisfied.

Let $(U(t), t \geq 0)$ be an admissible control, denoted generically as $U(t)=K(t) X(t)$, where $(X(t), t \geq 0)$ is the (unique) mild solution of (2.1) using the above admissible control. Let

$$
\mathcal{A}(t)=\left(a_{i j}(t)\right)
$$

and

$$
\tilde{\mathcal{A}}(t)=\left(\tilde{a}_{i j}(t)\right)
$$


where

$$
a_{i j}(t)=\int_{0}^{t}\left\langle\tilde{P} F_{i} X(s), \tilde{P} F_{j} X(s)\right\rangle d s
$$

if $B$ does not depend on $\alpha$ or

$$
a_{i j}(t)=\int_{0}^{t}\left\langle\tilde{P}\left(F_{i}+F_{i} \hat{B} K(s)\right) X(s), \tilde{P}\left(F_{j}+F_{j} \hat{B} K(s)\right) X(s)\right\rangle d s
$$

if $B(\alpha)$ has the form (2.12) and

$$
\tilde{a}_{i j}(t)=\frac{a_{i j}(t)}{a_{i i}(t)} .
$$

It is easy to verify that the integrations in (2.15a) and (2.15b) are well defined.

For the verification of the strong consistency of a family of least squares estimates of the unknown parameter vector, the following assumption is used.

(A8) For each admissible adaptive control law, $(\tilde{\mathcal{A}}(t), t \geq 0)$ satisfies

$$
\lim _{t \rightarrow \infty} \inf |\operatorname{det} \tilde{\mathcal{A}}(t)|>0 \text { a.s. }
$$

3. An Itô formula. In this section, an Itô formula is verified for a smooth function of the solution of (2.1). While some Itô formulas are available for evolution equations (e.g., [6], [15]), apparently no result is available for an equation of the form (2.1).

Since the parameter vector $\alpha$ is fixed in this section, the dependence of (2.1) on $\alpha$ is suppressed throughout this section. The Itô equation obtained here is verified by an approximation of (2.1) using the resolvent. For $\lambda>\beta$, let $R(\lambda)$ be defined by

$$
R(\lambda)=\lambda R(\lambda, A),
$$

where $R(\lambda, A)=(\lambda I-A)^{-1}$ is the resolvent of $A$. By assumptions (A2), $R(\lambda)^{1 / 2-\delta} \Phi$ is Hilbert-Schmidt and $R(\lambda) \Phi$ is Hilbert-Schmidt, so there is an $H$-valued Wiener process $\left(W_{\lambda}(t), t \geq 0\right)$ defined by

$$
W_{\lambda}(t)=W(t) \Phi^{*} R^{*}(\lambda)
$$

where $W_{\lambda}(1)$ has the nuclear covariance $R(\lambda) \Phi \Phi^{*} R^{*}(\lambda)$. Consider the stochastic differential equation

$$
\begin{aligned}
d X_{\lambda}(t) & =A X_{\lambda}(t) d t+R(\lambda) B U(t) d t+R(\lambda) d W_{\lambda}(t) \\
X_{\lambda}(0) & =R(\lambda) X_{0}
\end{aligned}
$$

where $\lambda>\beta$. It is shown that (3.3) has a strong solution.

LEMMA 3.1. For $\lambda>\beta$, the stochastic equation (3.3) has a unique strong solution on $[0, T]$, that is,

$$
\int_{0}^{T}\left|A X_{\lambda}(t)\right| d t<\infty \quad \text { a.s. }
$$

and

$$
\begin{aligned}
X_{\lambda}(t)= & R(\lambda) X_{0}+\int_{0}^{t} A X_{\lambda}(s) d s+\int_{0}^{t} R(\lambda) B U(s) d s \\
& +R(\lambda) W_{\lambda}(t) \quad \text { a.s. }
\end{aligned}
$$


Proof. To verify that the mild solution of (3.3) satisfies (3.5), it is necessary to show that

$$
\int_{0}^{T} \int_{0}^{t}|A S(t-s) R(\lambda) B U(s)| d s d t<\infty \quad \text { a.s. }
$$

and

$$
\int_{0}^{T} \int_{0}^{t}|A S(t-s) R(\lambda)|_{\mathcal{L}(H)}^{2} d s d t<\infty \quad \text { a.s. }
$$

We have

$$
\begin{aligned}
& |A S(t-s) R(\lambda) B|_{\mathcal{L}\left(H_{1}, H\right)} \\
& \leq|A S(t-s)|_{\mathcal{L}\left(D_{A}^{\varepsilon}, H\right)}|R(\lambda)|_{\mathcal{L}\left(D_{A}^{\varepsilon-1}, D_{A}^{\varepsilon}\right)}|B|_{\mathcal{L}\left(H_{1}, D_{A}^{\varepsilon-1}\right)} \\
& \leq \frac{c}{(t-s)^{1-\varepsilon}}
\end{aligned}
$$

so that

$$
\begin{aligned}
& \int_{0}^{T} \int_{0}^{t}|A S(t-s) R(\lambda) B U(s)| d s d t \\
& \leq T\left(\int_{0}^{T} \frac{c}{t^{q(1-\varepsilon)}} d t\right)^{1 / q}\left(\int_{0}^{T}|U(s)|^{p} d s\right)^{1 / p}
\end{aligned}
$$

which verifies (3.6). Since $|A S(t-s) R(\lambda)|_{\mathcal{L}(H)}$ is bounded for $0 \leq s \leq t \leq T$, (3.7) is satisfied. Use the Fubini theorem to compute $\int_{0}^{t} A X_{\lambda}(s) d s$ as in [6], [14] to verify (3.5).

Now it is shown that a sequence of processes can be obtained from solutions of (3.5) as $\lambda \rightarrow \infty$, which converges to (2.2).

LEMMA 3.2. There is a sequence $\left(\lambda_{n}\right)$ such that $\lambda_{n} \uparrow+\infty$, and, for $t \in[0, T]$,

$$
\lim _{n \rightarrow \infty} X_{\lambda_{n}}(t)=X(t) \quad \text { a.s. }
$$

and

$$
\sup \left\{\left|X_{\lambda_{n}}(t)\right|: \lambda_{n}>0, t \in[0, T]\right\}<\infty \text { a.s. }
$$

where $\left(X_{\lambda_{n}}(t), t \in[0, T]\right)$ satisfies (3.5) and $(X(t), t \in[0, T])$ satisfies $(2.1)$.

Proof. The Yosida approximation implies that $R(\lambda) X_{0} \rightarrow X_{0}$ as $\lambda \rightarrow \infty$ for all $X_{0} \in H$ and $|R(\lambda)|_{\mathcal{L}(H)} \leq c$ for some $c>0$. Since

$$
|S(t-s)(R(\lambda)-I) B U(s)| \leq \frac{K}{(t-s)^{1-\varepsilon}}|U(s)|,
$$

where $K>0$ is independent of $\lambda>\beta$, the Hölder inequality implies that

$$
\sup _{t \in[0, T]}\left|\int_{0}^{t} S(t-s) R(\lambda) B U(s) d s\right| \leq C_{T}\left(\int_{0}^{T}|U(s)|^{p} d s\right)^{1 / p}
$$

for some $C_{T}>0$ and the dominated convergence theorem implies that

$$
\lim _{\lambda \rightarrow \infty}\left|\int_{0}^{t} S(t-s)(R(\lambda)-I) B U(s) d s\right|=0
$$


for $t \in[0, T]$. Let $\bar{W}_{\lambda}(t)=W(t) \Phi^{*}\left(I-R^{2}(\lambda)\right)^{*}$, and, recalling (2.4), we have

$$
\begin{aligned}
& E \sup _{t \in[0, T]}\left|Z(t)-\int_{0}^{t} S(t-r) R(\lambda) d W_{\lambda}(r)\right|^{2} \\
& =E \sup _{t \in[0, T]}\left|\int_{0}^{t} S(t-r) d \bar{W}_{\lambda}(r)\right|^{2} .
\end{aligned}
$$

By Theorem 2.1 of [16], the right-hand side of (3.13) tends to zero as $\lambda \rightarrow \infty$ if the trace of the covariance operator of $(-A+\beta I)^{-(1 / 2)+\delta} W_{\lambda}(1)$ tends to zero as $\lambda \rightarrow \infty$. If $\left(e_{n}\right)$ is an orthonormal basis of $H$, then

$$
\begin{aligned}
& \operatorname{Tr}\left[(-A+\beta I)^{-(1 / 2)+\delta}\left(I-R^{2}(\lambda)\right) \Phi \Phi^{*}\left(I-R^{2}(\lambda)\right)^{*}\left(-A^{*}+\beta I\right)^{-(1 / 2)+\delta}\right] \\
& =\left|\left(I-R^{2}(\lambda)\right)(-A+\beta I)^{-(1 / 2)+\delta} \Phi\right|_{H S}^{2} \\
& =\sum_{n}\left|\left(I-R^{2}(\lambda)\right)(-A+\beta I)^{-(1 / 2)+\delta} \Phi e_{n}\right|^{2}
\end{aligned}
$$

This infinite series converges to zero as $\lambda \rightarrow \infty$ because

$$
\left|\left(I-R^{2}(\lambda)\right)(-A+\beta I)^{-(1 / 2)+\delta} \Phi e_{n}\right|^{2} \rightarrow 0
$$

for $n \in \mathbb{N}$, and the series is dominated by

$$
\sum_{n} 2\left(c^{4}+1\right)\left|(-A+\beta I)^{-(1 / 2)+\delta} \Phi e_{n}\right|=2\left(c^{4}+1\right)\left|(-A+\beta I)^{-(1 / 2)+\delta} \Phi\right|_{H S}^{2}<\infty .
$$

Thus the right-hand side of (3.13) converges to zero, and there is a sequence $\left(\lambda_{n}\right)$ such that

$$
\sup _{t \in[0, T]}\left|\int_{0}^{t} S(t-r) R\left(\lambda_{n}\right) d W_{\lambda_{n}}(t)-Z(t)\right|^{2} \rightarrow 0 \quad \text { a.s. }
$$

as $n \rightarrow \infty$. Therefore, for $t \in[0, T]$,

$$
\lim _{n \rightarrow \infty} X_{\lambda_{n}}(t)=X(t) \quad \text { a.s. }
$$

by (3.12) and (3.9) is satisfied because

$$
\sup _{\lambda>\beta, t \in[0, T]}\left|\int_{0}^{t} S(t-s) R(\lambda) B U(s) d s\right|<\infty \quad \text { a.s. }
$$

by (3.11).

An Itô formula is now verified for smooth functions of a solution of (2.1).

LEMMA 3.3. Let $V \in C^{1,2}([0, T] \times H, \mathbb{R})$ be such that $V_{x}(t, x) \in D_{A^{*}}^{1-\varepsilon}$ for all $t \in(0, T)$ and $V_{x}(t, \cdot): H \rightarrow D_{A^{*}}^{1-\varepsilon}$ is continuous. Assume that the function $\left\langle A x, V_{x}(t, x)\right\rangle$ for $x \in$ $\mathcal{D}(A)$ can be extended to a continuous function $h:[0, T] \times H \rightarrow \mathbb{R}$, the following limit exists:

$$
\lim _{\lambda \rightarrow \infty} \operatorname{Tr} V_{x x}(t, x) R^{2}(\lambda) \Phi \Phi^{*}\left(R^{*}(\lambda)\right)^{2}=\pi(t, x)<\infty
$$

and the map

$$
x \mapsto \operatorname{Tr} V_{x x}(t, x) R^{2}(\lambda) \Phi \Phi^{*}\left(R^{*}(\lambda)\right)^{2}
$$


is continuous on $H$ uniformly with respect to $\lambda \geq \beta$ and

$$
\begin{aligned}
|h(t, x)|+ & \left|\operatorname{Tr} V_{x x}(t, x) R^{2}(\lambda) \Phi \Phi^{*}\left(R^{*}(\lambda)\right)^{2}\right|+|V(t, x)| \\
& +\left|V_{x}(t, x)\right|_{D_{A^{*}}^{1-\varepsilon}}+\left|V_{x x}(t, x)\right| \mathcal{L}(H)+\left|V_{t}(t, x)\right| \leq k\left(1+|x|^{p}\right)
\end{aligned}
$$

for $(t, x) \in(0, T) \times H, \lambda \geq \beta$ where $k>0$ and $p>0$. Then

$$
\begin{aligned}
& V(t, X(t))-V(\tau, X(\tau)) \\
& =\int_{\tau}^{t}\left[h(s, X(s))+V_{s}(s, X(s))+\left\langle U(s), \Psi V_{x}(s, X(s))\right\rangle\right. \\
& \left.\quad+\frac{1}{2} \pi(s, X(s))\right] d s+\int_{\tau}^{t}\left\langle\Phi^{*} V_{x}(s, X(s)), d W(s)\right\rangle \quad \text { a.s. }
\end{aligned}
$$

where $0 \leq \tau \leq t \leq T, \Psi=B^{*}$ and $(X(t), t \in[0, T])$ satisfies $(2.1)$.

Proof. The verification of (3.18) is accomplished by using a sequence of processes that satisfy (3.5). Since $\left(X_{\lambda}(t), t \in[0, T]\right)$ is a strong solution of (3.5), the Itô formula [6] can be applied to $\left(V\left(X_{\lambda}(t)\right), t \in[0, T]\right)$ to obtain

$$
\begin{aligned}
V\left(t, X_{\lambda}(t)\right)-V\left(\tau, X_{\lambda}(\tau)\right)=\int_{\tau}^{t}[ & h\left(s, X_{\lambda}(s)\right)+V_{s}\left(s, X_{\lambda}(s)\right) \\
& +\left\langle U(s), \Psi R^{*}(\lambda) V_{x}\left(s, X_{\lambda}(s)\right)\right\rangle \\
& \left.+\frac{1}{2} \operatorname{Tr} R^{*}(\lambda) V_{x x}\left(s, X_{\lambda}(s)\right) R^{2}(\lambda) \Phi \Phi^{*} R^{*}(\lambda)\right] d s \\
& +\int_{\tau}^{t}\left\langle R^{*}(\lambda) V_{x}\left(s, X_{\lambda}(s)\right), d W_{\lambda}(s)\right\rangle \quad \text { a.s. }
\end{aligned}
$$

It suffices to assume that $(U(t), t \in[0, T])$ is uniformly bounded, almost surely. Lemma 3.2 verifies that

$$
\begin{aligned}
& \lim _{n \rightarrow \infty} V\left(t, X_{\lambda_{n}}(t)\right)=V(t, X(t)) \quad \text { a.s., } \\
& \lim _{n \rightarrow \infty} V_{s}\left(s, X_{\lambda_{n}}(s)\right)=V_{s}(s, X(s)) \quad \text { a.s., } \\
& \lim _{n \rightarrow \infty} V\left(\tau, X_{\lambda_{n}}(\tau)\right)=V(\tau, X(\tau)) \quad \text { a.s., } \\
& \lim _{n \rightarrow \infty} h\left(s, X_{\lambda_{n}}(s)\right)=h(s, X(s)) \quad \text { a.s. }
\end{aligned}
$$

for $0 \leq \tau \leq s \leq t \leq T$, where $\lambda_{n} \rightarrow \infty$. Let $\mathcal{L}_{\lambda} V$ and $\mathcal{L} V$ be defined as

$$
\begin{aligned}
\mathcal{L}_{\lambda} V(s, x)= & \left\langle U(s), \Psi R^{*}(\lambda) V_{x}(s, x)\right\rangle \\
& +\frac{1}{2} \operatorname{Tr} R^{*}(\lambda) V_{x x}(s, x) R^{2}(\lambda) \Phi \Phi^{*} R^{*}(\lambda)
\end{aligned}
$$

and

$$
\mathcal{L} V(s, x)=\left\langle U(s), \Psi V_{x}(s, x)\right\rangle+\frac{1}{2} \pi(s, x)
$$

for $(s, x) \in[0, T] \times H$. We have

$$
\begin{aligned}
& \left|\mathcal{L}_{\lambda} V\left(s, X_{\lambda}(s)\right)-\mathcal{L} V(s, X(s))\right| \\
& \leq\left|\mathcal{L}_{\lambda} V(s, X(s))-\mathcal{L} V(s, X(s))\right|+\left|\mathcal{L}_{\lambda} V(s, X(s))-\mathcal{L}_{\lambda} V\left(s, X_{\lambda}(s)\right)\right|
\end{aligned}
$$


From (3.15), we have that

$$
\lim _{\lambda \rightarrow \infty} \mathcal{L}_{\lambda} V(s, X(s))=\mathcal{L} V(s, X(s)) \quad \text { a.s. }
$$

Furthermore,

$$
\begin{aligned}
& \left|\mathcal{L}_{\lambda} V(s, x)-\mathcal{L}_{\lambda} V(s, y)\right| \\
& \leq|U(s)||\Psi|_{\mathcal{L}\left(D_{A^{*}}^{1-\varepsilon}, H_{1}\right)}\left|R^{*}(\lambda)\right|_{\mathcal{L}(H)}\left|V_{x}(s, x)-V_{x}(s, y)\right|_{D_{A^{*}}^{1-\varepsilon}} \\
& \quad+\frac{1}{2} \mid \operatorname{Tr} V_{x x}(s, x) R^{2}(\lambda) \Phi \Phi^{*}\left(R^{*}(\lambda)\right)^{2} \\
& \quad-\operatorname{Tr} V_{x x}(s, y) R^{2}(\lambda) \phi \phi^{*}\left(R^{*}(\lambda)\right)^{2} \mid
\end{aligned}
$$

The right-hand side of (3.26) tends to zero as $x \rightarrow y$ in $H$ uniformly with respect to $\lambda \geq \beta$ by (3.15). Thus the second term on the right-hand side of (3.24) converges to zero almost surely. Choosing a sequence $\left(\lambda_{n}\right)$ such that $\lambda_{n} \rightarrow \infty$, from Lemma 3.2 we obtain by (3.9), (3.17) and the dominated convergence theorem

$$
\begin{aligned}
& \lim _{n \rightarrow \infty} \int_{\tau}^{t}\left[h\left(s, X_{\lambda_{n}}(s)\right)+V_{s}\left(s, X_{\lambda_{n}}(s)\right)+\mathcal{L}_{\lambda_{n}} V\left(s, X_{\lambda_{n}}(s)\right) d s\right. \\
& =\int_{\tau}^{t}\left[h(s, X(s))+V_{s}(s, X(s))+\mathcal{L} V(s, X(s))\right] d s \quad \text { a.s. }
\end{aligned}
$$

Furthermore,

$$
\begin{aligned}
& E\left|\int_{\tau}^{t}\left\langle R^{*}\left(\lambda_{n}\right) V_{x}\left(s, X_{\lambda_{n}}(s)\right), d W_{\lambda_{n}}(s)\right\rangle-\int_{\tau}^{t}\left\langle\Phi^{*} V_{x}(s, X(s)), d W(s)\right\rangle\right|^{2} \\
& =E \int_{\tau}^{t}\left|\Phi^{*}\left(R^{*}\left(\lambda_{n}\right)\right)^{2} V_{x}\left(s, X_{\lambda_{n}}(s)\right)-\Phi^{*} V_{x}(s, X(s))\right|^{2} d s \\
& \leq 2 E \int_{\tau}^{t}\left[\mid \Phi^{*}\left(R^{*}\left(\lambda_{n}\right)\right)^{2}\left(V_{x}\left(s, X_{\lambda_{n}}(s)\right)-\left.V_{x}(s, X(s))\right|^{2}\right.\right. \\
& \left.\quad+\left|\left[\Phi^{*}\left(R^{*}(\lambda)\right)^{2}-\Phi^{*}\right] V_{x}(s, X(s))\right|^{2}\right] d s
\end{aligned}
$$

The right-hand side of (3.28) tends to zero as $n \rightarrow \infty$ by Lemma 3.2 and (3.17). Thus there is subsequence $\left(\lambda_{n_{j}}\right)$ such that

$$
\lim _{j \rightarrow \infty} \int_{\tau}^{t}\left\langle R^{*}\left(\lambda_{n_{j}}\right) V_{x}\left(s, X_{\lambda_{n_{j}}}(s)\right), d W_{\lambda_{n_{j}}}(s)\right\rangle=\int_{\tau}^{t}\left\langle\Phi^{*} V_{x}(s, X(s)), d W(s)\right\rangle \quad \text { a.s. }
$$

Thus (3.20)-(3.23), (3.27) verifies (3.18).

Now some of the hypotheses of Lemma 3.3 are replaced by ones that are more easily verified while still obtaining the same conclusion.

Proposition 3.4. Assume that (A2) is satisfied. Let $V \in C^{1,2}([0, T] \times H)$ be such that $V_{x}(t, x) \in D_{A^{*}}^{1-\varepsilon}, V_{x}(t, \cdot): H \rightarrow D_{A^{*}}^{1-\varepsilon}$ is continuous, $\left\langle A x, V_{x}(t, x)\right\rangle$ for $x \in \mathcal{D}(A)$ can be extended to a continuous function $h:[0, T] \times H \rightarrow \mathbb{R}$ and

$$
|h(t, x)|+|V(t, x)|+\left|V_{x}(t, x)\right|_{D_{A^{*}}^{1-\varepsilon}}+\left|V_{x x}(t, x)\right|_{\mathcal{L}(H)}+\left|V_{t}(t, x)\right| \leq k\left(1+|x|^{p}\right)
$$

for $(t, x) \in[0, T) \times H$ and $p>0, k>0$. Assume that one of the following three conditions is satisfied:

(i) $\Phi$ is Hilbert-Schmidt; 
(ii) $V_{x x}(t, x)$ is nuclear, $V_{x x}(t, \cdot)$ is continuous in the norm $|\cdot|_{1}$ of nuclear operators and $\left|V_{x x}(t, x)\right|_{1} \leq k\left(1+|x|^{p}\right)$ for $(t, x) \in(0, T) \times H$, where $k>0$ and $p>0$;

(iii) $V_{x x}(t, x) \in \mathcal{L}\left(D_{A}^{\delta-(1 / 2)}, D_{A^{*}}^{(1 / 2)-\delta}\right)$ for $(t, x) \in[0, T] \times H$, the function

$$
L(\cdot)=\left(R^{*}(\beta)\right)^{-(1 / 2)+\delta} V_{x x}(t, \cdot)(R(\beta))^{-(1 / 2)+\delta}: H \rightarrow \mathcal{L}(H)
$$

is continuous and $|L(x)|_{\mathcal{L}(H)} \leq k\left(1+|x|^{p}\right)$ is satisfied for $t>0$ and $x \in H$.

Then (3.18) is satisfied, where, for (i) and (ii), $\pi(t, x)=\operatorname{Tr} V_{x x}(t, x) \Phi \Phi^{*}$ and, for (iii), $\pi(t, x)=\operatorname{Tr}\left(R^{*}(\beta)\right)^{\delta-(1 / 2)} V_{x x}(t, x) \Phi \Phi^{*}\left(R^{*}(\beta)\right)^{(1 / 2)-\delta}$.

Proof. By Lemma 2.3, it suffices to show that (i), (ii), or (iii) implies (3.15)-(3.17). Assume that (i) is satisfied. Then

$$
\begin{aligned}
& \left|\operatorname{Tr}\left[V_{x x}(s, x) R^{2}(\lambda) \Phi \Phi^{*}(R(\lambda))^{2}-V_{x x}(s, x) \Phi \Phi^{*}\right]\right| \\
& =\left|\operatorname{Tr}\left[\left(R^{*}(\lambda)\right)^{2} V_{x x}(s, x) R^{2}(\lambda) \Phi \Phi^{*}-V_{x x}(s, x) \Phi \Phi^{*}\right]\right| \\
& \left.\leq \sum_{j} \mid\left(R^{*}(\lambda)\right)^{2} V_{x x}(s, x) R^{2}(\lambda)-V_{x x}(s, x)\right) \Phi \Phi^{*} e_{j} \mid,
\end{aligned}
$$

where $\left(e_{j}\right)$ is an orthonormal basis in $H$ that includes the eigenvectors of $\Phi \Phi^{*}$. The series on the right-hand side of (3.30) is dominated by

$$
\text { const } \sum_{j}\left|\Phi \Phi^{*} e_{j}\right|<\infty
$$

so the dominated convergence theorem implies that the series in (3.30) converges to zero as $\lambda \rightarrow \infty$. This verifies (3.15). Since

$$
\left|\operatorname{Tr}\left(V_{x x}(s, x)-V_{x x}(s, y)\right) R^{2}(\lambda) \Phi \Phi^{*}\left(R^{*}(\lambda)\right)^{2}\right| \leq c\left|V_{x x}(s, x)-V_{x x}(s, y)\right|_{\mathcal{L}(H)} \operatorname{Tr} \Phi \Phi^{*},
$$

where $c>0$ does not depend on $\lambda \geq \beta$, (3.16) is verified. Equation (3.17) follows from (3.29).

Assume that (ii) is satisfied. Then

$$
\begin{aligned}
& \left|\operatorname{Tr} V_{x x}(s, x) R^{2}(\lambda) \Phi \Phi^{*}\left(R^{*}(\lambda)\right)^{2}-\operatorname{Tr} V_{x x} \Phi \Phi^{*}\right| \\
& =\left|\operatorname{Tr}\left[R^{2}(\lambda) \Phi \Phi^{*}\left(R^{*}(\lambda)\right)^{2}-\Phi \Phi^{*}\right] V_{x x}(s, x)\right| \\
& \leq \sum_{j}\left|\left(R^{2}(\lambda) \Phi \Phi^{*}\left(R^{*}(\lambda)\right)^{2}-\Phi \Phi^{*}\right) V_{x x}(s, x) \hat{e}_{j}\right|,
\end{aligned}
$$

where $\left(\hat{e}_{j}\right)$ is an orthonormal basis in $H$ including the eigenvectors of $V_{x x}(s, x)$. Proceeding as for (i), it follows that (3.15) is satisfied. Since

$$
\begin{aligned}
& \left|\operatorname{Tr}\left(V_{x x}(s, x)-V_{x x}(s, y)\right) R^{2}(\lambda) \Phi \Phi^{*}\left(R^{*}(\lambda)\right)^{2}\right| \\
& \leq\left|R^{2}(\lambda) \Phi \Phi^{*}\left(R^{*}(\lambda)\right)^{2}\right|_{\mathcal{L}(H)}\left|V_{x x}(s, x)-V_{x x}(s, y)\right|_{1}, \\
& \quad\left|\operatorname{Tr} V_{x x}(s, x) R^{2}(\lambda) \Phi \Phi^{*}\left(R^{*}(\lambda)\right)^{2}\right| \\
& \leq k\left|R^{2}(\lambda) \Phi \Phi^{*}\left(R^{*}(\lambda)\right)^{2}\right|_{\mathcal{L}(H)}\left(1+|x|^{p}\right),
\end{aligned}
$$

so (3.16), (3.17) are satisfied.

Assume that (iii) is satisfied. Then

$$
\begin{aligned}
& \left|\operatorname{Tr} V_{x x}(s, x) R^{2}(\lambda) \Phi \Phi^{*}\left(R^{*}(\lambda)\right)^{2}-\pi(s, x)\right| \\
& =\mid \operatorname{Tr}\left[\left(R^{*}(\lambda)\right)^{2} L(x) R^{2}(\lambda) R^{(1 / 2)-\delta}(\beta) \Phi \Phi^{*}\left(R^{*}(\beta)\right)^{(1 / 2)-\delta}\right. \\
& \left.\quad-L(x) R^{(1 / 2)-\delta}(\beta) \Phi \Phi^{*}(R(\beta))^{(1 / 2)-\delta}\right] \mid .
\end{aligned}
$$


Since the operator $R^{(1 / 2)-\delta}(\beta) \Phi \Phi^{*}\left(R^{*}(\beta)\right)^{(1 / 2)-\delta}$ is nuclear by (A2), we can choose an orthonormal basis in $H$ that includes the eigenvectors of this operator and proceed as in (i) to verify (3.15). Since

$$
\begin{aligned}
& \left|\operatorname{Tr} V_{x x}(s, x) R^{2}(\lambda) \Phi \Phi^{*}\left(R^{*}(\lambda)\right)^{2}-\operatorname{Tr} V_{x x}(s, y) R^{2}(\lambda) \Phi \Phi^{*}\left(R^{*}(\lambda)\right)^{2}\right| \\
& \leq\left|\left(R^{*}(\lambda)\right)^{2}(L(x)-L(y)) R^{2}(\lambda)\right|_{\mathcal{L}(H)}\left|R^{(1 / 2)-\delta}(\beta) \Phi \Phi^{*}\left(R^{*}(\beta)\right)^{(1 / 2)-\delta}\right|_{1}
\end{aligned}
$$

and

$$
\begin{aligned}
& \left|\operatorname{Tr} V_{x x}(s, x) R^{2}(\lambda) \Phi \Phi^{*}\left(R^{*}(\lambda)\right)^{2}\right| \\
& \leq\left|\left(R^{*}(\lambda)\right)^{2} L(x) R^{2}(\lambda)\right|_{\mathcal{L}(H)} \cdot\left|R^{(1 / 2)-\delta}(\beta) \Phi \Phi^{*}\left(R^{*}(\beta)\right)^{(1 / 2)-\delta}\right|_{1} \\
& \leq \operatorname{const}\left(1+|x|^{p}\right)
\end{aligned}
$$

these inequalities verify (3.16), (3.17).

For use of this Itô formula in the adaptive control problem, it is useful to state explicitly the case where $v(x)=\langle V x, x\rangle$, where $V \in \mathcal{L}(H)$ is a selfadjoint operator.

COROLlaRY 3.5. Let $V \in \mathcal{L}(H)$ be selfadjoint such that $V \in \mathcal{L}\left(H, D_{A^{*}}^{1-\varepsilon}\right)$ and $|\langle V x, A x\rangle| \leq k|x|^{2}$ for $x \in \mathcal{D}(A)$, where $k>0$. Assume that one of the following conditions is satisfied:

(i) $\Phi$ is Hilbert-Schmidt,

(ii) $V$ is nuclear,

(iii) $V \in \mathcal{L}\left(D_{A}^{\delta-(1 / 2)}, D_{A^{*}}^{(1 / 2)-\delta}\right)$.

Then, for all $0 \leq \tau \leq t \leq T$,

$$
\begin{aligned}
& \langle V X(t), X(t)\rangle-\langle V X(\tau), X(\tau)\rangle \\
& =\int_{\tau}^{t}[h(X(s))+2\langle U(s), \Psi V X(s)\rangle+\Pi(V)] d s \\
& \quad+2 \int_{\tau}^{t}\left\langle\Phi^{*} V X(s), d W(s)\right\rangle \quad \text { a.s., }
\end{aligned}
$$

where $h$ is the continuous extension of $2\langle V x, A x\rangle$ on $H$, and, for (i) and (ii), $\Pi(V)=$ $\operatorname{Tr} V \Phi \Phi^{*}$ and, for (iii), $\Pi(V)=\operatorname{Tr}\left(R^{*}(\beta)\right)^{\delta-(1 / 2)} V \Phi \Phi^{*}\left(R^{*}(\beta)\right)^{(1 / 2)-\delta}$.

4. Parameter identification. For the identification of the unknown parameters in the linear stochastic distributed parameter system (2.1), a family of least squares estimates are formed. In this section, it is assumed that $\beta=0$, that is, $-A(\alpha)$ is strictly positive. Let $\tilde{P}$ be the projection given in (A7). The estimate of the unknown parameter vector at time $t, \hat{\alpha}(t)$ is the minimizer of the quadratic functional of $\alpha, L(t ; \alpha)$, given by

$$
\begin{aligned}
L(t ; \alpha)=- & \int_{0}^{t}\langle\tilde{P}(A(\alpha)+B(\alpha) K(s)) X(s), d \tilde{P} X(s)\rangle \\
& +\frac{1}{2} \int_{0}^{t}|\tilde{P}(A(\alpha)+B(\alpha) K(s)) X(s)|^{2} d s,
\end{aligned}
$$

where $U(s)=K(s) X(s)$ is an admissible adaptive control.

THEOREM 4.1. Let $(K(t), t \geq 0)$ be an admissible feedback control law. Assume that (A2), (A6)-(A8) are satisfied and $\alpha_{0} \in \mathcal{K}^{0}$. Then the family of least squares estimates $(\hat{\alpha}(t), t>0)$, where $\hat{\alpha}(t)$ is the minimizer of (4.1), is strongly consistent, that is,

$$
P_{\alpha_{0}}\left(\lim _{t \rightarrow \infty} \hat{\alpha}(t)=\alpha_{0}\right)=1,
$$

where $\alpha_{0}$ is the true parameter vector. 
Proof. If $B$ does not depend on $\alpha \in \mathcal{K}$, then the proof of Theorem 4.1 follows from the proof of Theorem 1 in [10]. Therefore we may assume that $B(\alpha)$ has the form (2.12). Since the strong law of large numbers for Brownian motion is used to verify strong consistency, it is shown initially that

$$
\lim _{t \rightarrow \infty} \int_{0}^{t}|\langle\ell, X(s)\rangle|^{2} d s=+\infty \quad \text { a.s. }
$$

for suitable $\ell \in H$.

If $\left(P_{n}\right)$ is a sequence of increasing finite-dimensional projections with range in $\cap_{i=0}^{p} \mathcal{D}\left(F_{i}^{*}\right)$ that converges strongly to the identity $I$ and $F \in\left\{F_{1}, \ldots, F_{q}\right\}$, then

$$
\begin{aligned}
& \lim _{n \rightarrow \infty} \int_{0}^{t}\left\langle\tilde{P} F(I+\hat{B} K(s)) P_{n} X(s), \tilde{P} F(I+\hat{B} K(s)) P_{n} X(s)\right\rangle d s \\
& =\int_{0}^{t}\langle\tilde{P} F(I+\hat{B} K(s)) X(s), \tilde{P} F(I+\hat{B} K(s)) X(s)\rangle d s
\end{aligned}
$$

in $L^{1}(P)$ almost surely because the sequence of integrals is monotone increasing.

Fix $n \in \mathbb{N}$. For the process $\left(P_{n} X(t), t \geq 0\right)$ with nonzero values in a finite-dimensional space, the verification of (4.3) is accomplished by using some of the methods in [9] for finitedimensional systems. Since the expectation of the Lebesgue measure of the amount of time that a scalar Brownian motion is strictly away from zero is infinite, the 0-1 law for Brownian motion implies that, for almost all sample paths, the Lebesgue measure of the amount of time that a sample path is strictly away from zero is infinite.

If $\ell \in H$ and $\Phi^{*} \ell \neq 0$, then a well-known property of a scalar Brownian motion implies that

$$
\liminf _{t \rightarrow \infty}\left\langle\Phi^{*} \ell, W(t)\right\rangle=-\infty \quad \text { a.s. }
$$

and

$$
\limsup _{t \rightarrow \infty}\left\langle\Phi^{*} \ell, W(t)\right\rangle=+\infty \quad \text { a.s. }
$$

Fix $n \in \mathbb{N}$ and $\ell \in \cap_{i=0}^{q} \mathcal{D}\left(F_{i}^{*}\right)$ such that $\Phi^{*} \ell \neq 0$ and $P_{n} \ell \neq 0$. Assume that $P_{n} \tilde{P}=\tilde{P}$. Let $\left(T_{n}\right)$ be a sequence of stopping times such that $\left\{\lim _{n \rightarrow \infty}\left\langle\Phi^{*} \ell, W\left(T_{n}\right)\right\rangle=+\infty\right\}$. Let $\Lambda_{+}=$ $\left\{\lim \sup _{n \rightarrow \infty} \int_{0}^{T_{n}}\left\langle M^{*}(s) \ell, X(s)\right\rangle d s=+\infty\right\}$, where $M^{*}(s)=\left(A^{*}+K^{*}(s) \hat{B}^{*} A^{*}\right) P_{n}$. For each $\omega \in \Lambda_{+}$, there is a subsequence $\left(T_{n_{j}}(\omega)\right)$ such that

$$
\lim _{j \rightarrow \infty} \int_{0}^{T_{n_{j}}(\omega)}\left\langle M^{*}(s, \omega) \ell, X(s, \omega)\right\rangle d s=+\infty .
$$

Since

$$
\begin{aligned}
\langle\ell, X(t)\rangle^{2} & \geq\left(\int_{0}^{t}\left\langle M^{*}(s) \ell, X(s)\right\rangle d s\right)^{2} \\
& +2 \int_{0}^{t}\left\langle M^{*}(s) \ell, X(s)\right\rangle d s\left\langle\Phi^{*} \ell, W(t)\right\rangle \\
& +\left\langle\Phi^{*} \ell, W(t)\right\rangle^{2},
\end{aligned}
$$

it follows directly that, for almost all $\omega \in \Lambda_{+}$,

$$
\limsup _{t \rightarrow \infty}\langle\ell, X(t, \omega)\rangle^{2}=+\infty .
$$


Let $\Lambda_{-}=\left\{\lim \sup _{n \rightarrow \infty} \int_{0}^{T_{n}}\left\langle M^{*}(s) \ell, X(s)\right\rangle d s=-\infty\right\}$. For $\omega \in \Lambda_{-}$,

$$
\lim _{n \rightarrow \infty} \int_{0}^{T_{n}}\left\langle M^{*}(s, \omega) \ell, X(s, \omega)\right\rangle d s=-\infty
$$

Since $-W$ has the same probability law as $W$, we have that, for almost $\omega \in \Lambda_{-}$,

$$
\limsup _{t \rightarrow \infty}\langle\ell, X(t, \omega)\rangle^{2}=+\infty .
$$

Let $\Lambda_{0}=\left\{\lim \sup _{n \rightarrow \infty}\left|\int_{0}^{T_{n}}\left\langle M^{*}(s) \ell, X(s)\right\rangle d s\right|<\infty\right\}$. It follows immediately that, for almost all $\omega \in \Lambda_{0}$,

$$
\limsup _{t \rightarrow \infty}\langle\ell, X(t, \omega)\rangle^{2}=+\infty
$$

Combining the results of the above three cases, we have

$$
\limsup _{t \rightarrow \infty}\langle\ell, X(t)\rangle^{2}=+\infty \quad \text { a.s. }
$$

Since the Lebesgue measure of the amount of time that $\left(\left\langle\ell, P_{n} X(t)\right\rangle, t \geq 0\right)$ is strictly away from zero is infinite for almost all sample paths, it follows that

$$
\lim _{t \rightarrow \infty} \int_{0}^{t}|\langle\ell, X(s)\rangle|^{2} d s=+\infty \quad \text { a.s. }
$$

By (A7), it follows that there is a $\bar{c}>0$ such that

$$
\operatorname{Tr}\left[\left(\hat{P}\left(I+K^{*}(s) \hat{B}^{*}\right) F^{*} \tilde{P}\right)^{*}\left(\hat{P}\left(I+K^{*}(s) \hat{B}^{*}\right) F^{*} \tilde{P}\right)\right]>c,
$$

for all $s \in \mathbb{R}_{+}$, and $F \in\left\{F_{1}, \ldots, F_{q}\right\}$, which implies that

$$
\lim _{t \rightarrow \infty} \int_{0}^{t}\left\langle\hat{P}\left(I+K^{*}(s) \hat{B}^{*}\right) F^{*} \tilde{P} X(s), \hat{P}\left(I+K^{*}(s) \hat{B}^{*}\right) F^{*} \tilde{P} X(s)\right\rangle d s=+\infty \quad \text { a.s. }
$$

and, consequently,

$$
\lim _{t \rightarrow \infty} \int_{0}^{t}\langle\tilde{P} F(I+\hat{B} K(s)) X(s), \tilde{P} F(I+\hat{B} K(s)) X(s)\rangle d s=+\infty \quad \text { a.s. }
$$

To minimize (4.1) with respect to $\alpha$, it is necessary and sufficient that $D_{\alpha} L(t ; \alpha)=0$. Computing the family of partial derivatives and using (2.1), we obtain the family of linear equations

$$
\mathcal{A}(t) \hat{\alpha}(t)=\mathcal{A}(t) \alpha_{0}+b(t)
$$

or

$$
\tilde{\mathcal{A}}(t) \hat{\alpha}(t)=\tilde{\mathcal{A}}(t) \alpha_{0}+\tilde{b}(t)
$$

where $\mathcal{A}(t)$ and $\tilde{\mathcal{A}}(t)$ are given by (2.13) and (2.14), respectively, and

$$
b_{j}(t)=\int_{0}^{t}\left\langle\tilde{P}\left(F_{j}+\hat{B} K(s)\right) X(s), d \tilde{P} \Phi W(s)\right\rangle,
$$




$$
\begin{gathered}
\tilde{b}_{j}(t)=\frac{b_{j}(t)}{a_{j j}(t)}, \\
b(t)=\left(b_{1}(t), \ldots, b_{q}(t)\right)^{\prime}, \\
\tilde{b}(t)=\left(\tilde{b}_{1}(t), \ldots, \tilde{b}_{q}(t)\right)^{\prime} .
\end{gathered}
$$

Let $\left(c_{n}\right)$ be a sequence of positive, real numbers such that $c_{n} \downarrow 0$. Let $\Lambda_{n}=$ $\left\{\liminf _{t \rightarrow \infty}|\operatorname{det} \tilde{\mathcal{A}}(t)|>c_{n}>0\right\}$. The sequence $\left(\Lambda_{n}\right)$ is increasing. By (A8), we have that $P\left(\Lambda_{n}\right) \uparrow 1$ as $n \rightarrow \infty$. Given $\varepsilon>0$, there is an $N \in \mathbb{N}$ such that $P\left(\Lambda_{N}\right)>1-\varepsilon$. There is a random time such that $|\operatorname{det} \tilde{\mathcal{A}}(t, \omega)|>c_{n}$ for $\omega \in \Lambda_{N}$ and $t \geq T(\omega)$. Since $\tilde{b}(t) \rightarrow 0$ almost surely as $t \rightarrow \infty$ by the strong law of large numbers for Brownian motion, since $\tilde{\mathcal{A}}^{*}(t) \tilde{\mathcal{A}}(t)$ is uniformly bounded almost surely, and since $\varepsilon>0$ is arbitrary, it follows that $\hat{\alpha}(t) \rightarrow \alpha_{0}$ almost surely as $t \rightarrow \infty$.

For the applications of identification and adaptive control, it is important to have recursive estimators of the unknown parameters. Let $\langle\tilde{F}(s) x, y\rangle$ be the vector whose $i$ th component is $\left\langle\tilde{P} F_{i}(I+\hat{B} K(s)) x, y\right\rangle$. Using (2.1), (4.9), we have

$$
\hat{\alpha}(t)=\mathcal{A}^{-1}(t) \int_{0}^{t}\left\langle\tilde{F}(s) X(s), d \tilde{P} X(s)-\tilde{P} F_{0} X(s) d s\right\rangle .
$$

Since $\mathcal{A}^{-1}(t)$ satisfies the differential equation

$$
d \mathcal{A}^{-1}(t)=-\mathcal{A}^{-1}(t) d \mathcal{A}(t) \mathcal{A}^{-1}(t)
$$

the differential of (4.11) satisfies

$$
d \hat{\alpha}(t)=\mathcal{A}^{-1}(t)\langle\tilde{F}(t) X(t), d \tilde{P} X(s)-\tilde{P} A(\hat{\alpha}(t))(I+\hat{B} K(t)) X(t) d t\rangle .
$$

5. Optimality for an adaptive control. In this section, the certainty equivalence, optimal ergodic control law is shown to be self-tuning and self-optimizing. The self-tuning property is obtained by using the continuity of the solution of a stationary Riccati equation with respect to parameters in the topology induced by a suitable operator norm. Since the unbounded operator $B(\alpha)$ appears in the linear transformation of the control in (2.1), this operator topology is more restrictive than for bounded linear transformations on the Hilbert space. This continuity property is also used to show that the certainty equivalence control stabilizes the unknown system in a suitable sense. The self-optimizing property is verified for this adaptive control.

The solution $V$ of the stationary Riccati equation (2.9) satisfies the assumptions of Corollary 3.5 if one of the following three conditions is satisfied: (i) $\Phi$ is Hilbert-Schmidt, (ii) $V$ is nuclear, or (iii) $A$ is strictly negative. By (A5), $V \in \mathcal{L}\left(H, D_{A^{*}}^{1-\varepsilon}\right)$ (see [12], [18]), and (2.10) implies that

$$
|\langle A x, V x\rangle|=|\langle R x, x\rangle| \leq k|x|^{2}
$$

for some $R \in \mathcal{L}(H)$. If $A$ is strictly negative, then it easily follows that

$$
V \in \mathcal{L}\left(D_{A}^{\delta-(1 / 2)}, D_{A^{*}}^{(1 / 2)-\delta}\right) .
$$

Moreover, if (A2) is satisfied with $\Phi=I$, then $V$ is nuclear because, from Theorems 1 and 2 of [12], it follows that $P_{a}=\left(-A^{*}+\beta I\right)^{a} V \in \mathcal{L}(H)$ for each $a \in(0,1)$. Thus 
$V=P_{a}^{*}(-A+\beta I)^{-a}$ is nuclear because $(-A+\beta I)^{-a}$ is nuclear for $a=1-2 \delta$ by (A2), (A5).

If an adaptive control is self-tuning and some stability properties are satisfied for the solution of (2.1), then this adaptive control is self-optimizing.

PROPOSITION 5.1. Assume that (A2), (A5) are satisfied, that the solution $V$ of (2.10) satisfies the assumptions of Corollary 3.5, and that

$$
\begin{aligned}
\lim _{t \rightarrow \infty} \frac{1}{t}\langle V X(t), X(t)\rangle=0 \quad \text { a.s. } \\
\limsup _{t \rightarrow \infty} \frac{1}{t} \int_{0}^{t}|X(s)|^{2} d s<\infty \quad \text { a.s. }
\end{aligned}
$$

where $(X(t), t \geq 0)$ is the solution of (2.1) with $\alpha_{0} \in \mathcal{K}$ and the control $U \in$ $\cap_{T>0} M_{W}^{p}\left(0, T, H_{1}\right)$. Then

$$
\liminf _{T \rightarrow \infty} \frac{1}{T} J\left(X_{0}, U, \alpha_{0}, T\right) \geq \Pi(V) \text { a.s. }
$$

where $V$ is the solution of (2.10) with $\alpha=\alpha_{0}$. Furthermore, if $U$ is an admissible control $U(t)=K(t) X(t)$ such that

$$
\lim _{t \rightarrow \infty} K(t)=k_{0} \quad \text { a.s. }
$$

in the uniform $\mathcal{L}\left(H, H_{1}\right)$ topology where $k_{0}=-P^{-1} \Psi V$, then

$$
\lim _{T \rightarrow \infty} \frac{1}{T} J\left(X_{0}, U, \alpha_{0}, T\right)=\Pi(V) \text { a.s. }
$$

Proof. For $U \in M_{W}^{p}\left(0, T, H_{1}\right)$, we have

$$
\begin{aligned}
\langle V X(t), X(t)\rangle-\langle V x, x\rangle= & \int_{0}^{t} h(X(s))+2\langle U(s), \Psi V X(s)\rangle d s \\
& +t \Pi(V)+2 \int_{0}^{t}\left\langle\Phi^{*} V X(s), d W(s)\right\rangle \quad \text { a.s. }
\end{aligned}
$$

where $h(x)$ is the continuous extension of $2\langle A x, V x\rangle, x \in \mathcal{D}(A)$. Using the stationary Riccati equation (2.10), we obtain

$$
\begin{aligned}
& \langle V X(t), X(t)\rangle-\left\langle V X_{0}, X_{0}\right\rangle \\
& =\int_{0}^{t}\left[2\langle U(s), \Psi V X(s)\rangle+\left\langle P^{-1} \Psi V X(s), \Psi V X(s)\right\rangle-\langle Q X(s), X(s)\rangle\right] d s \\
& \quad+2 \int_{0}^{t}\left\langle\Phi^{*} V X(s), d W(s)\right\rangle+t \Pi(V) \text { a.s. }
\end{aligned}
$$

By a similar method as in Proposition 2 of [10], we obtain from (5.2) that

$$
\lim _{t \rightarrow \infty} \frac{1}{t} \int_{0}^{t}\left\langle\Phi^{*} V X(s), d W(s)\right\rangle=0 \quad \text { a.s. }
$$

Thus

$$
\lim _{T \rightarrow \infty}\left(\frac{1}{T} J\left(X_{0}, U, \alpha_{0}, T\right)-\frac{1}{T} \int_{0}^{T}\left|P^{1 / 2} U(s)+P^{-1} \Psi V X(s)\right|^{2} d s\right)=\Pi(V) \quad \text { a.s. }
$$


and (5.3) is verified. If $U(t)=K(t) X(t)$ and if (5.4) is satisfied, then

$$
\lim _{T \rightarrow \infty} \frac{1}{T} \int_{0}^{T}\left|P^{1 / 2}\left(U(s)+P^{-1} \Psi V X(s)\right)\right|^{2} d s=0 \quad \text { a.s. }
$$

by (5.3). Thus (5.5) is verified.

Now it is shown that the stability conditions (5.1), (5.2) are satisfied for an admissible, self-tuning adaptive control.

PROPOSITION 5.2. Assume that (A2), (A5) are satisfied. Let the solution $V$ of (2.10) satisfy the assumptions of Corollary 3.5. If $(X(t), t \geq 0)$ is the solution of (2.1) with $\alpha_{0} \in \mathcal{K}$ and an adaptive control law $(K(t), t \geq 0)$ that satisfies (5.4), then (5.1), (5.2) are satisfied.

Proof. Apply the Itô formula (3.31) of Corollary 3.5 to $\langle V X(t), X(t)\rangle$ again to obtain (5.6). Let $(P(t), t \geq 0)$ satisfy

$$
\begin{aligned}
P(t)= & \int_{0}^{t}[h(X(s))+2\langle K(s) X(s), \Psi V X(s)\rangle \\
& \left.+\frac{1}{2}\langle Q X(s), X(s)\rangle\right] d s+2 \int_{0}^{t}\left\langle\Phi^{*} V X(s), d W(s)\right\rangle
\end{aligned}
$$

and use the stationary Riccati equation (2.10) to obtain

$$
\begin{aligned}
P(t)= & \int_{0}^{t}\left[2\langle K(s) X(s), \Psi V X(s)\rangle+2\left\langle P^{-1} \Psi V X(s), \Psi V X(s)\right\rangle\right. \\
& \left.-\left\langle P^{-1} \Psi V X(s), \Psi V X(s)\right\rangle-\frac{1}{2}\langle Q X(s), X(s)\rangle\right] d s \\
& +2 \int_{0}^{t}\left\langle\Phi^{*} V X(s), d W(s)\right\rangle .
\end{aligned}
$$

By (2.7), (2.8) and the boundedness of $\Phi^{*} V$, there are constants $c_{0}, c_{1}, c_{2}$, and $c_{3}$ such that

$$
\begin{aligned}
P(t) \leq & \int_{0}^{t}\left[2\left\langle\left(P^{-1} \Psi V+K(s)\right) X(s), \Psi V X(s)\right\rangle-c_{0}|X(s)|^{2}\right] d s+2 \int_{0}^{t}\left\langle\Phi^{*} V X(s), d W(s)\right\rangle \\
\leq & \int_{0}^{t}\left(-c_{1}+c_{2}\left|K(s)-k_{0}\right| \mathcal{L}\left(H, H_{1}\right)\right)|X(s)|^{2} d s \\
& \quad+\int_{0}^{t}\left|\Phi^{*} V X(s)\right|^{2} d s\left[-c_{3}+\frac{2}{\int_{0}^{t}\left|\Phi^{*} V X(s)\right|^{2} d s} \int_{0}^{t}\left\langle\Phi^{*} V X(s), d W(s)\right\rangle\right] \\
= & P_{1}(t)+P_{2}(t) .
\end{aligned}
$$

Since $K(s) \rightarrow k_{0}$ almost surely as $s \rightarrow \infty, \lim _{\sup _{t \rightarrow \infty}}(1 / t) P_{1}(t) \leq 0$ almost surely, and by the strong law of large numbers for Brownian motion, we have $\lim \sup _{t \rightarrow \infty}(1 / t) P_{2}(t) \leq 0$ almost surely. Thus

$$
\Pi(V) \geq \limsup _{t \rightarrow \infty}\left[\frac{1}{t}\langle V X(t), X(t)\rangle+\frac{1}{2 t} \int_{0}^{t}\langle Q X(s), X(s)\rangle d s\right] \quad \text { a.s., }
$$

and (5.2) is verified. 
To verify (5.1), again use the Itô formula (Corollary 3.5) for $\langle V X(t), X(t)\rangle$ as

$$
\begin{aligned}
\langle & V X(t), X(t)\rangle-\langle V X(\tau), X(\tau)\rangle \\
= & \int_{\tau}^{t}\left[2\langle K(s) X(s), \Psi V X(s)\rangle-2\left\langle k_{0} X(s), \Psi V X(s)\right\rangle\right. \\
& -\langle Q X(s), X(s)\rangle+\Pi(V)] d s+2 \int_{\tau}^{t}\left\langle\Phi^{*} V X(s), d W(s)\right\rangle \\
\leq & \int_{\tau}^{t}\left(-c_{1}+c_{2}\left|K(s)-k_{0}\right|\right)|X(s)|^{2} d s+(t-\tau) \Pi(V) \\
& +2 \int_{\tau}^{t}\left\langle\Phi^{*} V X(s), d W(s)\right\rangle,
\end{aligned}
$$

where $c_{1}>0, c_{2}>0$ and $\tau \in[0, t]$. Thus

$$
\begin{aligned}
& \langle V X(t), X(t)\rangle-\langle V X(\tau), X(\tau)\rangle \\
& \leq \int_{\tau}^{t}-c_{3}\langle V X(s), X(s)\rangle d s+c_{4}(t-\tau)+M(\tau, t)
\end{aligned}
$$

for some $c_{3}>0, c_{4}>0$, and $t \geq \tau>T_{0}$, where $T_{0}$ is a random time and where

$$
M(\tau, t)=2 \int_{\tau}^{t}\left\langle\Phi^{*} V X(s), d W(s)\right\rangle .
$$

Let $\psi(t)=\langle V X(t), X(t)\rangle$ and let $\left(y(t), t \geq T_{0}\right)$ satisfy

$$
y(t)=\psi\left(T_{0}\right)-c_{3} \int_{T_{0}}^{t} y(s) d s+c_{4}\left(t-T_{0}\right)+M\left(T_{0}, t\right)
$$

for $t \geq T_{0}$. Taking the difference of (5.8) and (5.9), it is clear that $\psi(t) \leq y(t)$ almost surely for $t \geq T_{0}$. Solving the integral equation (5.9), we have

$$
\begin{aligned}
y(t)= & \psi\left(T_{0}\right) e^{-c_{3}\left(t-T_{0}\right)}+\frac{c_{4}}{c_{3}}\left(1-e^{-c_{3}\left(t-T_{0}\right)}\right) \\
& -c_{3} \int_{T_{0}}^{t} e^{-c_{3}(t-s)} M\left(T_{0}, s\right) d s+M\left(T_{0}, t\right) \quad \text { a.s. }
\end{aligned}
$$

From (5.2), which has been verified above, and from the strong law of large numbers for Brownian motion, we have that

$$
\lim _{t \rightarrow \infty} \frac{1}{t} y(t)=0 \quad \text { a.s. }
$$

which verifies (5.1).

To verify the self-tuning property for the certainty equivalence adaptive control, $K(t)=$ $-P^{-1} \Psi(\hat{\alpha}(t-\Delta)) V(\hat{\alpha}(t-\Delta))$, where $(\hat{\alpha}(t), t \geq 0)$ is a family of strongly consistent estimators of the true parameter vector $\alpha_{0}$. It is important to show a suitable continuous dependence of the solution $V(\alpha)$ of the stationary Riccati equation on $\alpha \in \mathcal{K}$. For $B$ bounded, some results are given in [5], [10]. For $B$ unbounded, as in (2.1), we can use a continuity result from [17, Thms. 1.1 and 5.3], which is reformulated below. It is assumed that $A(\alpha)$ is strictly negative for each $\alpha \in \mathcal{K}$. For notational convenience, let $A_{0}=A\left(\alpha_{0}\right)$, where $\alpha_{0}=\mathcal{K}$ is the true parameter value. 
LEMMA 5.3. Assume that (A1), (A3), (A4) are satisfied and that

$$
\begin{aligned}
\lim _{\alpha \rightarrow \alpha_{0}}\left|\Psi(\alpha)-\Psi\left(\alpha_{0}\right)\right|_{\mathcal{L}\left(D_{A_{0}^{*}}^{1-\varepsilon}, H_{1}\right)} & =0, \\
\lim _{\alpha \rightarrow \alpha_{0}}\left|S(t ; \alpha)-S\left(t ; \alpha_{0}\right)\right|_{\mathcal{L}\left(D_{A_{0}}^{\varepsilon-1}, H\right)} & =0
\end{aligned}
$$

for each $t \geq 0$, where $\Psi(\alpha)=B^{*}(\alpha)$. Then

$$
\lim _{\alpha \rightarrow \alpha_{0}}\left|V(\alpha)-V\left(\alpha_{0}\right)\right|_{\mathcal{L}\left(H, D_{A_{0}^{*}}^{1-\varepsilon}\right)}=0 .
$$

Note that (A3) and (5.11) imply that

$$
\lim _{\alpha \rightarrow \alpha_{0}}\left|A^{-1}(\alpha)-A^{-1}\left(\alpha_{0}\right)\right|_{\mathcal{L}(H)}=0,
$$

and, from (A1), (5.10) and (5.11), we have that

$$
\lim _{\alpha \rightarrow \alpha_{0}}\left|\Psi(\alpha) S^{*}(t ; \alpha)-\Psi\left(\alpha_{0}\right) S^{*}\left(t ; \alpha_{0}\right)\right|_{\mathcal{L}\left(H, H_{1}\right)}=0 .
$$

Thus we can follow the proof of Theorem 5.3 in [17] to obtain (5.12).

The self-optimizing property is now verified for a self-tuning adaptive control.

THEOREM 5.4. Assume that (A1)-(A4), (A6)-(A8) are satisfied. Let $(\hat{\alpha}(t), t \geq 0)$ be the family of least squares estimates, where $\hat{\alpha}(t)$ is the minimizer of $(4.1)$. Let $(K(t), t \geq 0)$ be an admissible adaptive control law such that

$$
K(t)=-P^{-1} \Psi(\hat{\alpha}(t-\Delta)) V(\hat{\alpha}(t-\Delta)),
$$

where $\Psi(\alpha)=B^{*}(\alpha)$ and $V(\alpha)$ is the solution of (2.10) for $\alpha \in \mathcal{K}$. Then the family of estimates $(\hat{\alpha}(t), t \geq 0)$ is strongly consistent,

$$
\lim _{t \rightarrow \infty} K(t)=k_{0} \quad \text { a.s. }
$$

in $\mathcal{L}\left(H, H_{1}\right)$ where $k_{0}=-P^{-1} \Psi\left(\alpha_{0}\right) V\left(\alpha_{0}\right)$, and

$$
\lim _{T \rightarrow \infty} \frac{1}{T} J\left(X_{0}, U, \alpha_{0}, T\right)=\operatorname{Tr} \Pi\left(V\left(\alpha_{0}\right)\right) \text { a.s. }
$$

where $U(t)=K(t) X(t)$ and $\Pi(V)$ is given in Corollary 3.5 .

The proof follows directly from Theorem 4.1, Lemma 5.3, and Propositions 5.1 and 5.2 with $A=A\left(\alpha_{0}\right)$. The solution $V=V\left(\alpha_{0}\right)$ of the Riccati equation satisfies the assumptions of Corollary 3.5 because $A\left(\alpha_{0}\right)$ is strictly negative.

\section{Some examples.}

Example 6.1. This is a family of examples from elliptic differential operators. Let $G$ be a bounded, open domain in $\mathbb{R}^{n}$ with $C^{\infty}$-boundary $\partial G$ with $G$ locally on one side of $\partial G$ and let $L(x, D)$ be an elliptic differential operator of the form

$$
L(x, D) f=\sum_{i, j=1}^{n} D_{i} a_{i j}(x) D_{j} f+\sum_{i=1}^{n}\left[b_{i}(x) D_{i} f+D_{i}\left(d_{i}(x) f\right)\right]+c(x) f
$$

where the coefficients $a_{i j}, b_{i}, d_{i}, c$ are elements of $C^{\infty}(\bar{G})$,

$$
\sum_{i, j} a_{i j}(x) \xi_{i} \xi_{j} \geq \hat{\nu}|\xi|^{2}
$$


where $\xi=\left(\xi_{1}, \ldots, \xi_{n}\right) \in \mathbb{R}^{n}, x \in G, \hat{\nu}>0$ is a constant, and $\left\{a_{i j}(x)\right\}$ is symmetric. Consider a stochastic parabolic control problem formally described by the equations

$$
\frac{\partial y}{\partial t}(t, x)=L(x, D) y(t, x)+\eta(t, x)
$$

for $(t, x) \in \mathbb{R}_{+} \times G$ and

$$
\frac{\partial y}{\partial \nu}(t, x)+h(x) y(t, x)=u(t, x)
$$

for $(t, x) \in \mathbb{R}_{+} \times \partial G$ and $y(0, x)=y_{0}(x)$, where $\partial / \partial \nu=\sum_{i, j=1}^{n} a_{i j} \nu_{j} D_{i}$ is the normal derivative, $\nu=\left(\nu_{1}, \ldots, \nu_{n}\right)$ is the outward normal to $\partial G$, the process $(\eta(t, x) ;(t, x) \in$ $\left.\mathbb{R}_{+} \times G\right)$ formally denotes a space dependent white noise, $u \in L^{2}\left(0, T, L^{2}(\partial G)\right)$ for any $T>0, h \in C^{\infty}(\partial G)$, and $h \geq 0$.

To give a precise meaning to (6.3), (6.4), the semigroup approach is used. An intuitive justification of the semigroup model (2.1) is given. Let $H=L^{2}(G), H_{1}=L^{2}(\partial G)$ and define $A f=L(x, D) f . A: H \rightarrow H$ is densely defined, and $\mathcal{D}(A)=\left\{f \in H^{2}(G)\right.$ : $\partial f / \partial \nu+h f=0$ on $\partial G\}$. It is well known that $A$ generates an analytic semigroup, the linear operator $(A-\beta I)$ is strictly negative for some $\beta \geq 0$.

To introduce the control operator, consider the elliptic problem

$$
(L(x, D)-\beta) z=0 \quad \text { on } G,
$$

$$
\frac{\partial z}{\partial \nu}+h z=-g \text { on } \partial G
$$

For $g \in L^{2}(\partial G)$, there is a unique solution $z \in H^{3 / 2}(G)$ [19]. Define $\hat{B} \in \mathcal{L}\left(H_{1}, H^{3 / 2}(G)\right)$ by the equation $\hat{B} g=-z$. For $\varepsilon<3 / 4$, we have $\hat{B} \in \mathcal{L}\left(H_{1}, D_{A}^{\varepsilon}\right)$ because $D_{A}^{(3 / 4)-\gamma}=$ $H^{(3 / 2)-2 \gamma}$ for any sufficiently small $\gamma>0$ [13]. Let $y_{\beta}(t, x)=e^{-\beta t} y(t, x)$ and $\eta(t, x) d t=$ $\Phi d W(t)$ for some $\Phi \in \mathcal{L}(H)$ and a cylindrical Wiener process $(W(t), t \geq 0)$ in $H$. From (6.5), (6.6), we have

$$
\begin{gathered}
d y_{\beta}=(L(x, D)-\beta) y_{\beta} d t+e^{-\beta t} \Phi d W(t), \\
\frac{\partial y_{\beta}}{\partial \nu}+h y_{\beta}=e^{-\beta t} u=u_{\beta}(t) \quad \text { on } \partial G \\
y_{\beta}(0)=y(0)
\end{gathered}
$$

Formally performing the differentiation $(\partial / \partial t) \hat{B} u_{\beta}(t)$, we obtain

$$
\begin{gathered}
d \omega_{\beta}(t)=\left((L(x, D)-\beta) y_{\beta}(t)-\hat{B} \dot{u}_{\beta}(t)\right) d t+e^{-\beta t} \Phi d W(t) \\
\frac{\partial \omega_{\beta}}{\partial \nu}+h \omega_{\beta}=0 \quad \text { on } \mathbb{R}_{+} \times \partial G
\end{gathered}
$$

where $\omega_{\beta}(t)=y_{\beta}(t)-\hat{B} u_{\beta}(t)$. For (6.7), the formula for the mild solution is

$$
\begin{aligned}
\omega_{\beta}(t)= & S_{\beta}(t)(y(0)+\hat{B} u(0))+\int_{0}^{t} S_{\beta}(t-r) \Phi e^{\beta r} d W(r) \\
& -\int_{0}^{t} S_{\beta}(t-r) \hat{B} \dot{u}_{\beta}(r) d r
\end{aligned}
$$


where $S_{\beta}(t)=e^{t(A-\beta I)}$. Formally integrating by parts the last integral in (6.9) yields

$$
\begin{aligned}
y_{\beta}(t)=\omega_{\beta}(t)-\hat{B} u_{\beta}(t)= & S_{\beta}(t) y(0)+\int_{0}^{t}(A-\beta I) S_{\beta}(t-r) \hat{B} u_{\beta}(r) d r \\
& +\int_{0}^{t} e^{-\beta r} S_{\beta}(t-r) \Phi d W(r) .
\end{aligned}
$$

Thus, cancelling $e^{-\beta t}$, we have

$$
y(t)=S(t) y(0)+\int_{0}^{t} S(t-r) B u(r) d r+\int_{0}^{t} S(t-r) \Phi d W(r),
$$

which is a mild solution of the form (2.3), where $B=\Psi^{*}$ and $\Psi \in \mathcal{L}\left(D_{A^{*}}^{1-\varepsilon}, H_{1}\right)$ extends the operator $\hat{B}^{*}\left(A^{*}-\beta I\right)$.

The assumptions that are used in this paper are now verified for this example. Assumption (A2) may not be satisfied so that it can be considered as a condition on the noise term (specifically on $\Phi$ ). If $\Phi$ is Hilbert-Schmidt or if $\Phi W(t)$ evolves in $H$, then (A2) is satisfied. If $n=1$ and $\Phi \in \mathcal{L}(H)$, then (A2) is satisfied. In this case,

$$
A f=\frac{\partial}{\partial x} a(x) \frac{\partial}{\partial x} f+\left(b(x) \frac{\partial}{\partial x} f+\frac{\partial}{\partial x} d(x) f+c(x) f\right) .
$$

Let $A_{1}=(\partial / \partial x) a(x)(\partial / \partial x)$. By Corollary 2.6.11 of [20],

$$
\left|\left(-A_{1}^{*}+\beta I\right)^{\gamma} x\right|^{2} \leq \mathrm{const}\left|\left(-A^{*}+\beta I\right)^{\gamma} x\right|^{2}
$$

for $x \in \mathcal{D}\left(\left(\beta I-A^{*}\right)^{\gamma}\right)$, where $\gamma \in\left(0, \frac{1}{2}\right)$. It follows that $(-A+\beta I)^{-\gamma}$ is Hilbert-Schmidt if $\left(-A_{1}+\beta I\right)^{-\gamma}$ is Hilbert-Schmidt. Since $A_{1}=A_{1}^{*}$ and $\left(A_{1}-\beta I\right)^{-1}$ is compact, we can use the comments following Lemma 2.1 to conclude that $\left(-A_{1}+\beta I\right)^{-(1 / 2)+\delta}$ is Hilbert-Schmidt if and only if

$$
\int_{0}^{T} t^{-2 \delta}|\tilde{S}(t)|_{H S}^{2} d t<\infty
$$

for $T>0$, where $(\tilde{S}(t), t \geq 0)$ is the semigroup generated by $A_{1}-\beta I$. We have

$$
|\tilde{S}(t)|_{H S}^{2}=\int_{G} \int_{G}|G(t, \theta, r)|^{2} d r d \theta
$$

where $G(t, \theta, r)$ is the Green function for the problem

$$
\frac{\partial \omega}{\partial t}=\left[a(x) \omega^{\prime}\right]^{\prime}-\beta \omega, \quad \frac{\partial \omega}{\partial \nu}+h \omega=0,
$$

since

$$
|G(t, \theta, r)| \leq \frac{k_{1}}{\sqrt{t}} \exp \left[k_{2} \frac{|\theta-r|^{2}}{t}\right]
$$

for $t>0$ and $\theta, r \in G$, where $k_{1}$ and $k_{2}$ are positive constants [1], [2]. Condition (6.10) is satisfied for any $\delta \in\left(0, \frac{1}{4}\right)$. Thus (A2) is satisfied for any $\Phi \in \mathcal{L}(H)$. Assumption (A5) can be shown to be satisfied. For example, this is trivially satisfied if the operator $A$ is strictly negative. In the above example, if $A\left(=A\left(\alpha_{0}\right)\right)$ is strictly negative and (A2) is satisfied, then, 
for the control system (2.1) with $\alpha=\alpha_{0}$ and the cost functional (2.6) where $Q \in \mathcal{L}\left(L^{2}(G)\right)$ and $P \in \mathcal{L}\left(L^{2}(\partial G)\right)$ are uniformly positive, the self-optimizing property (5.5) of Proposition 5.1 is satisfied.

Now consider a parameter dependent version of (6.3), (6.4)

$$
\begin{gathered}
\frac{\partial y}{\partial t}(t, x)=\alpha L(x, D) y(t ; x)+\eta(t, x), \quad(t, x) \in \mathbb{R}_{+} \times G, \\
\frac{\partial y}{\partial \nu}=u(t, x), \quad(t, x) \in \mathbb{R}_{+} \times \partial G, \\
y(0, x)=y_{0}(x),
\end{gathered}
$$

where $\alpha \in \mathcal{K}=\left[\alpha_{1}, \alpha_{2}\right]$ is scalar parameter for $0<\alpha_{1}<\alpha_{2}$. Assume that the operator $A$ corresponding to $L(x, D)$ is strictly negative and that (A2) is satisfied. Using the same semigroup model as above, we have that

$$
\begin{aligned}
y(t ; \alpha)= & S(t ; \alpha) y_{0}+\alpha \int_{0}^{t} S(t-r ; \alpha) B U(r) d r \\
& +\int_{0}^{t} S(t-r ; \alpha) \Phi d W(r),
\end{aligned}
$$

where $S(t ; \alpha)=e^{t \alpha A}, B=\left[\hat{B}^{*} A^{*}\right]^{*} \in \mathcal{L}\left(D_{A^{*}}^{1-\varepsilon}, H_{1}\right)$ and $\hat{B} \in \mathcal{L}\left(H_{1}, D_{A}^{\varepsilon}\right)$ solves the elliptic problem

$$
\begin{gathered}
L(x, D)(\hat{B} g)=0 \quad \text { on } G \\
\frac{\partial}{\partial \nu}(\hat{B} g)=-g \quad \text { on } \partial G .
\end{gathered}
$$

Assumptions (A1), (A3)-(A6), and (5.10) are now trivially satisfied because $A(\alpha)=\alpha A, \alpha \in$ $\left[\alpha_{1}, \alpha_{2}\right], \alpha_{1}>0$, and $A$ is strictly negative. Condition (5.11) is satisfied because $S(\cdot) \in$ $C\left((0, \infty), D_{A}^{1-\varepsilon}\right)$. Furthermore, we have that $\left(A^{*}\right)^{-1} \in \mathcal{L}(H), \hat{B} \in \mathcal{L}\left(H_{1}, D_{A}^{\varepsilon}\right)$ and that the embedding $D_{A}^{\varepsilon} \rightarrow H$ is compact, so (A7) is satisfied. Since the parameter is scalar, (A8) is trivially satisfied. Thus, by Theorem 4.1 , the family of least squares estimates given in the statement there is strongly consistent for $\alpha_{0} \in\left(\alpha_{1}, \alpha_{2}\right)$. For any strongly consistent family of estimators $(\hat{\alpha}(t), t \geq 0)$, the cost functional (2.6) with a uniformly positive $Q \in \mathcal{L}\left(L^{2}(G)\right)$ and $P \in \mathcal{L}\left(L^{2}(\partial G)\right)$, system (2.1) with $A\left(\alpha_{0}\right)=\alpha_{0} A, B$ as above, $\beta=0$, the adaptive control

$$
U(t)=-P^{-1} \Psi(\hat{\alpha}(t-\Delta)) V(\hat{\alpha}(t-\Delta)) X(t)
$$

has the self-optimizing property (5.15) by Theorem 5.4 .

An elementary example of a boundary control problem with a vector parameter $\alpha$ is described that satisfies (A8). It is a specialization of (6.5). Let $H=L^{2}([0,1], \mathbb{R})$, let $F_{1}$ and $F_{2}$ be the linear operators

$$
F_{1}=\frac{d^{2}}{d x^{2}}, \quad F_{2}=\frac{d}{d x}
$$

and let $A(\alpha)$ be

$$
A(\alpha)=\alpha_{1} F_{1}+\alpha_{2} F_{2}
$$


where $\alpha=\left(\alpha_{1}, \alpha_{2}\right), \alpha_{i} \in\left[\bar{a}_{i}, \bar{b}_{i}\right]$ and $\bar{a}_{i}>0, \bar{b}_{i}<\infty$ for $i=1,2$. The domain of $A(\alpha)$ is $\mathcal{D}(A(\alpha))=\left\{f \in H^{2}(0,1): \partial f / \partial \nu=0\right.$ on $\left.\{0,1\}\right\}$. Let $\left(k_{n}, \ell_{n} ; n=0,1, \ldots\right)$ be the basis of $H$, defined as

$$
\begin{aligned}
& k_{n}(x)=\sqrt{2} \sin 2 n \pi x, \\
& \ell_{n}(x)=\sqrt{2} \cos 2 n \pi x .
\end{aligned}
$$

Fix a positive integer $N$ and let $\tilde{P}$ be the projection determined by the family $\left(k_{n}, \ell_{n}, n=\right.$ $1,2, \ldots, N)$. Since the adaptive control law $(B K(t), t \geq 0)$ is a family of compact operators, it is the limit of a family of finite rank operators. Thus, to evaluate $\mathcal{A}(t)$, it suffices to apply $F_{1}$ and $F_{2}$ to the finite sum $\sum_{j=1}^{\tilde{N}}\left(a_{j} k_{j}+b_{j} \ell_{j}\right)$. It is elementary to verify that

$$
\left\langle F_{1} k_{n}, F_{2} k_{m}\right\rangle=\left\langle F_{1} \ell_{n}, F_{2} \ell_{m}\right\rangle=0
$$

for all $m$ and $n$ and that

$$
\left\langle F_{1} k_{n}, F_{2} \ell_{m}\right\rangle=\left\langle F_{1} \ell_{n}, F_{2} k_{m}\right\rangle=0
$$

for $n \neq m$. Thus

$$
\begin{aligned}
& \left\langle F_{1} \sum_{j=1}^{\tilde{N}}\left(a_{j} k_{j}+b_{j} \ell_{j}\right), F_{2} \sum_{j=1}^{\tilde{N}}\left(a_{j} k_{j}+b_{j} \ell_{j}\right)\right\rangle \\
& =\sum_{j=1}^{\tilde{N}} a_{j} b_{j}\left(\left\langle F_{1} k_{j}, F_{2} \ell_{j}\right\rangle+\left\langle F_{1} \ell_{j}, F_{2} k_{j}\right\rangle\right) \text { a.s. }
\end{aligned}
$$

Since we have

$$
\left\langle F_{1} k_{j}, F_{2} \ell_{j}\right\rangle=(2 j \pi)^{3}, \quad\left\langle F_{1} \ell_{j}, F_{2} k_{j}\right\rangle=-(2 j \pi)^{3},
$$

by passage to the limit and integration, it follows that

$$
a_{12}(t)=0 \quad \text { a.s. }
$$

for $t>0$, and the matrix $\mathcal{A}(t)$ is diagonal. Thus, for $t>0, \tilde{\mathcal{A}}(t)=I$ almost surely and $\operatorname{det} \tilde{\mathcal{A}}(t)=1$ almost surely; so (A8) is trivially satisfied. This example can be generalized to many space dimensions. For example, consider the dimension- 2 case. Let $H=L^{2}([0,1] \times$ $[0,1], \mathbb{R})$ and let $F_{1}, F_{2}$, and $F_{3}$ be the linear operators

$$
F_{1}=\frac{\partial^{2}}{\partial x^{2}}+\frac{\partial^{2}}{\partial y^{2}}, \quad F_{2}=\frac{\partial}{\partial x}, \quad F_{3}=\frac{\partial}{\partial y} .
$$

Let $A(\alpha)$ be

$$
A(\alpha)=\alpha_{1} F_{1}+\alpha_{2} F_{2}+\alpha_{3} F_{3}
$$

where $\alpha=\left(\alpha_{1}, \alpha_{2}, \alpha_{3}\right), \alpha_{i} \in\left[\tilde{a}_{i}, \tilde{b}_{i}\right], \tilde{a}_{i}>0$, and $\tilde{b}_{i}<\infty$ for $i=1,2,3$. It easily follows by computations that are similar to the above that the matrix $\mathcal{A}(t)$ is diagonal, so that, for $t>0, \tilde{\mathcal{A}}(t)=I$ almost surely and $\operatorname{det} \tilde{\mathcal{A}}(t)=1$ almost surely. Thus (A8) is satisfied. 
Example 6.2. This example is a structurally damped plate with random loading and point control. Consider the following model of a plate in the deflection $w$ :

$$
w_{t t}(t, x)+\Delta^{2} w(t, x)-\alpha \Delta w(t, x)=\delta\left(x-x_{0}\right) u(t)+\eta(t, x)
$$

for $(t, x) \in \mathbb{R}_{+} \times G$,

$$
\begin{gathered}
w(0, \cdot)=w_{0}, \quad w_{t}(0, \cdot)=w_{1}, \\
\left.w\right|_{\mathbb{R}_{+} \times \partial G}=\left.\Delta w\right|_{\mathbb{R}_{+} \times \partial G}=0,
\end{gathered}
$$

where $\alpha>0$ is an unknown constant, $\eta(t, x)$ formally represents a space-dependent Gaussian white noise on the open, bounded, smooth domain $G \subset \mathbb{R}^{n}$ for $n \leq 3$, and $\delta\left(x-x_{0}\right)$ is the Dirac distribution at $x_{0} \in G$. The cost functional is

$$
J\left(w_{0}, w_{1}, u, \alpha, T\right)=\int_{0}^{T}\left(|w(t)|_{H^{2}(G)}^{2}+\left|w_{t}(t)\right|_{L^{2}(G)}^{2}+|u(t)|^{2}\right) d t .
$$

For a mathematical treatment of the deterministic problem (6.14)-(6.17) where $\eta \equiv 0$, refer to [3], [4], [17] and references therein. Define the linear operator $\mathcal{A}$ by the equation $\mathcal{A} h=\Delta^{2} h$, where $\mathcal{D}(\mathcal{A})=\left\{h \in H^{4}(G):\left.h\right|_{\partial G}=\left.\Delta h\right|_{\partial G}=0\right\}$. Following [4], [17], (6.14)-(6.17) are rewritten in the form (2.1), (2.6), where $H=\mathcal{D}\left(\mathcal{A}^{1 / 2}\right) \times L^{2}(G)=\left(H^{2}(G) \cap H_{0}^{1}(G)\right) \times$ $L^{2}(G), H_{1}=\mathbb{R}$,

$$
\begin{gathered}
A(\alpha)=\left[\begin{array}{cc}
0 & I \\
-\mathcal{A} & -\alpha \mathcal{A}^{1 / 2}
\end{array}\right], \\
B u=\left[\begin{array}{c}
0 \\
\delta\left(x-x_{0}\right) u
\end{array}\right], \\
\Phi=\left[\begin{array}{cc}
0 & 0 \\
0 & \Phi_{1}
\end{array}\right],
\end{gathered}
$$

where $\Phi_{1} \in \mathcal{L}\left(L^{2}(G)\right)$ is a Hilbert-Schmidt operator and where $\Phi_{1} \Phi_{1}^{*}>0, Q=I, P=I$, and $(W(t), t \geq 0)$ in (2.1) is a cylindrical Wiener process on $H$. It is known [4] that $A(\alpha)$ generates a stable analytic semigroup, $(S(t ; \alpha), t \geq 0)$, and that $B \in \mathcal{L}\left(H_{1}, D_{A(\alpha)}^{\varepsilon-1}\right)$ for $\varepsilon \in(0,1-n / 4)$, which is possible for $n \leq 3$ (cf. [17]). Suppose that the unknown parameter $\alpha \in \mathcal{K}=\left[a_{0}, a_{1}\right]$, where $0<a_{0}<a_{1}$. Assumptions (A1), (A2), (A4)-(A6) are clearly satisfied. Since $B$ does not depend on $\alpha \in \mathcal{K}$, assumption (A7) is satisfied with a finitedimensional projection $\tilde{P}: H \rightarrow \tilde{P}(H)$ of the form

$$
\tilde{P}=\left[\begin{array}{cc}
0 & 0 \\
0 & \tilde{P}_{1}
\end{array}\right]
$$

where $\tilde{P}_{1}: L^{2}(G) \rightarrow H^{2}(G)$ and $\tilde{P}_{1} \neq 0$. Assumption (A8) is trivially satisfied because the parameter $\alpha$ is scalar. The assumptions of the uniform analyticity and the exponential stability of the semigroup $(S(t ; \alpha), t \geq 0)$ and the continuous dependence of this semigroup on $\alpha,(5.11)$, can be verified by the explicit spectral expansions of $A(\alpha)$ and $(S(t ; \alpha), t \geq 0)$ [4, Thm. A3]. Therefore, by Theorem 4.1, the family of least squares estimates given in the statement there is strongly consistent for $\alpha_{0} \in\left(a_{0}, a_{1}\right)$. For any strongly consistent family of estimators $(\hat{\alpha}(t), t \geq 0)$, system (2.1) with $A\left(\alpha_{0}\right), B, \Phi$ as above, $\beta=0$, and the adaptive control $(U(t), t \geq 0)$ given by

$$
U(t)=-\Psi(\hat{\alpha}(t-\Delta)) V(\hat{\alpha}(t-\Delta)) X(t)
$$

has the self-optimizing property $(5.15)$ by Theorem 5.4 . 
Acknowledgments. We thank A. Bensoussan for suggesting the study of adaptive boundary control problems, and we dedicate this paper to him on his fiftieth birthday, when this work was initiated, following his suggestion. We also thank I. Vrkoc and J. Seidler for reading the manuscript and suggesting improvements, and I. Lasiecka for providing important information on deterministic, distributed parameter systems that broadened the scope of this paper.

\section{REFERENCES}

[1] H. Amann, On abstract parabolic fundamental solutions, J. Math. Soc. Japan, 39 (1987), pp. 93-116.

[2] R. ARIMA, On general boundary value problem for parabolic equations, J. Math. Kyoto Univ., 4 (1964), pp. 207-243.

[3] G. CHEN AND D. L. Russell, A mathematical model for linear elastic systems with structural damping, Quart. Appl. Math., 39 (1982), pp. 433-454.

[4] S. Chen And R. Triggiani, Proof of extensions of two conjectures on structural damping for elastic systems, Pacific J. Math., 136 (1989), pp. 15-55.

[5] A. Chojnowska-Michalik, T. E. DunCAN, And B. Pasik-DunCan, Uniform operator continuity of the stationary Riccati equation in Hilbert space, Appl. Math. Optim., 25 (1992), pp. 171-187.

[6] R. CuRTAin AND P. FALB, Itô's lemma in infinite dimensions, J. Math. Anal. Appl., 31 (1970), pp. $434-448$.

[7] G. DAPRATO, S. KWAPIEN, AND J. ZABCZYK, Regularity of solutions of linear stochastic equations in Hilbert spaces, Stochastics, 23 (1987), pp. 1-23.

[8] G. DaPrato and A. ICHIKaWa, Riccati equations with unbounded coefficients, Ann. Mat. Pura Appl., 140 (1985), pp. 209-221.

[9] T. E. Duncan and B. Pasik-Duncan, Adaptive control of continuous time linear stochastic systems, Math. Control Signals Systems, 3 (1990), pp. 45-60.

[10] T. E. Duncan, B. Goldys, And B. Pasik-Duncan, Adaptive control of linear stochastic evolution systems, Stochastics and Stochastic Reports, 35 (1991), pp. 129-142.

[11] F. FLANDOLI, Direct solution of a Riccati equation arising in a stochastic control problem with control and observations on the boundary, J. Appl. Math. Optim., 14 (1986), pp. 107-129.

[12] - Algebraic Riccati equation arising in boundary control problems, SIAM J. Control Optim., 25 (1987), pp. 612-636.

[13] D. FuJIWARA, Concrete characterizations of the domains of fractional powers of some elliptic differential operators of the second order, Proc. Japan Acad. Ser. A. Math. Sci., 43 (1967), pp. 82-86.

[14] A. ICHIKAWA, Stability of semilinear stochastic evolution equations, J. Math. Anal. Appl., 190 (1982), pp. $12-44$.

[15] - Semilinear stochastic evolution equations: Boundedness, stability and invariant measures, Stochastics, 12 (1984), pp. 1-39.

[16] P. Kotelenez, A maximal inequality of stochastic convolution integrals on Hilbert spaces and space-time regularity of linear stochastic partial differential equations, Stochastics, 21 (1987), pp. 345-358.

[17] I. LASIECKA AND R. TRIGGIANI, Numerical approximations of algebraic Riccati equations modelled by analytic semigroups and applications, Math. Comput., 57 (1991), pp. 639-662, 513-537.

[18] - The regulator problem for parabolic equations with Dirichlet boundary control I, Appl. Math. Optim., 16 (1987), pp. 147-168.

[19] J. L. Lions And E. Magenes, Nonhomogeneous Boundary Value Problems and Applications I, Springer-Verlag, Berlin, 1972.

[20] A. PAZY, Semigroups of Linear Operators and Applications to Partial Differential Equations, Springer-Verlag, New York, 1983. 